\title{
Unimolecular pyrolysis mechanism of thiophene and furan: an ab initio comparative study
}

\author{
Tianshuang $\mathrm{Li}^{1,2}, \mathrm{Jie} \mathrm{Li}^{1,2}$, Hongliang Zhang ${ }^{1,2 *}$, Jingkun Wang ${ }^{1,2}$, Jin Xiao ${ }^{1,2}$ \\ 1 School of Metallurgy and Environment, Central South University, Hunan Province, \\ Changsha 410083, China. \\ 2 National Engineering Laboratory of High Efficient Recovery of Refractory \\ Nonferrous Metals, Central South University, Hunan Province, Changsha 410083, \\ China.
}

\section{Supporting Information}




\section{CONTENT}

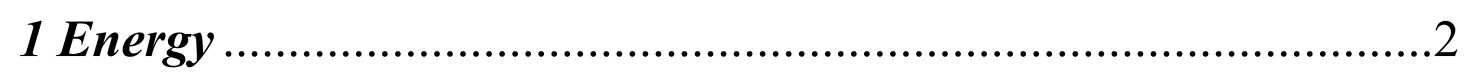

2 Bond Rotaion .....................................................................

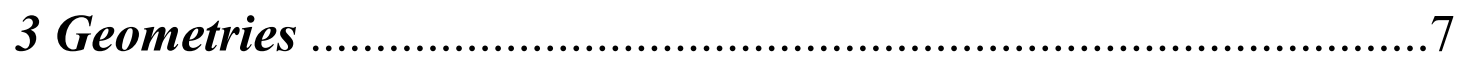

2.1 Geometries in Section 3.1 ................................................

2.2 Geometries in Section 3.2 ................................................. 10

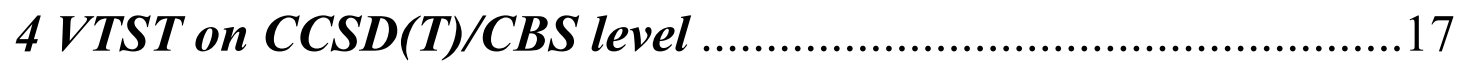




\section{Energy}

All the IMs and TSes have been computed (optimization and frequency calculation) at M062x/def2-QZVP, CBS-QB3, G4, CBS-APNO (only for furan) and W1BD level.

Table S1 and Table S2 presents the potential energy and the Gibbs free energy of thiophene IMs and TSes, respectively. Table S3 and Table S4 illustrate the potential energy and the Gibbs free energy of furan IMs and TSes, respectively.

Table S1 The potential energy $(0 \mathrm{~K})$ of thiophene intermediates and transition states at different levels. (Unit: $\mathrm{kcal} / \mathrm{mol}$ )

\begin{tabular}{|c|c|c|c|c|c|c|}
\hline & $\begin{array}{l}\text { B3LYP/ } \\
\text { cc-pVTZ }\end{array}$ & $\begin{array}{l}\text { M062x/ } \\
\text { def2qzvp }\end{array}$ & CBS-QB3 & G4 & $\begin{array}{c}\mathrm{CCSD}(\mathrm{T}) \\
/ \mathrm{CBS}\end{array}$ & W1BD \\
\hline $\mathrm{C}_{2,4}$ & 76.07 & 77.27 & 76.21 & 74.97 & 75.44 & 75.64 \\
\hline $\mathrm{C}_{2,5}$ & 54.22 & 56.18 & 55.57 & 54.55 & 54.48 & 54.91 \\
\hline TS- $\mathrm{C}_{2,3}-\mathrm{C}_{2,4}$ & 86.99 & 86.20 & 85.91 & 85.98 & 86.05 & 86.34 \\
\hline TS- $\mathrm{C}_{2,4}-\mathrm{C}_{2,5}$ & 87.46 & 86.75 & 87.78 & 87.00 & 86.88 & 87.28 \\
\hline TS- $\mathrm{C}_{2,5}-\mathrm{C}_{2,1}$ & 94.66 & 94.94 & 94.41 & 93.83 & 93.77 & 93.86 \\
\hline IM1 & 39.85 & 46.39 & 46.91 & 46.48 & 47.20 & 47.90 \\
\hline IM2 & 50.29 & 52.70 & 52.28 & 51.61 & 52.17 & 52.87 \\
\hline IM3 & 37.51 & 39.67 & 39.20 & 38.62 & 38.78 & 39.16 \\
\hline IM4 & 50.94 & 52.74 & 53.64 & 52.93 & 53.70 & 53.96 \\
\hline IM5 & 49.33 & 47.64 & 50.98 & 50.16 & 50.49 & 50.98 \\
\hline IM6 & 30.32 & 36.22 & 36.84 & 36.19 & 37.57 & 38.00 \\
\hline IM7 & 89.44 & 98.73 & 88.67 & 88.52 & 90.61 & 91.61 \\
\hline IM8 & 76.11 & 44.43 & 81.35 & 80.32 & 81.55 & 82.87 \\
\hline IM9 & 57.37 & 59.00 & 61.59 & 60.84 & 61.44 & 61.82 \\
\hline IM10 & 40.88 & 43.48 & 45.63 & 45.10 & 44.76 & 45.35 \\
\hline IM11 & 102.13 & 109.93 & 112.32 & 111.66 & 110.31 & 111.52 \\
\hline BIM6 & 82.26 & 90.39 & 92.66 & 91.75 & 92.53 & 93.04 \\
\hline BIM7 & 74.78 & 96.18 & 86.15 & 98.77 & 88.03 & 87.99 \\
\hline TS1 & 96.50 & 102.69 & 99.40 & 99.74 & 101.15 & 101.87 \\
\hline TS2 & 144.46 & 150.60 & 147.31 & 146.74 & 147.56 & 148.81 \\
\hline TS3 & 92.88 & 92.52 & 91.49 & 91.21 & 91.71 & 92.42 \\
\hline TS4 & 61.19 & 69.07 & 67.17 & 67.14 & 68.10 & 68.99 \\
\hline TS5 & 94.62 & 95.65 & 96.89 & 95.58 & 95.91 & 97.14 \\
\hline TS6 & 95.36 & 98.15 & 96.36 & 96.05 & 96.72 & 97.24 \\
\hline TS7 & 122.18 & 133.06 & 129.44 & 128.76 & 131.20 & 132.51 \\
\hline TS8 & 119.30 & 126.50 & 119.87 & 119.26 & 122.83 & 125.23 \\
\hline TS9 & 109.57 & 117.69 & 112.43 & 112.10 & 113.23 & 114.50 \\
\hline TS10 & 119.24 & 130.17 & 124.82 & 124.03 & 125.54 & 126.48 \\
\hline TS11 & 124.69 & 139.53 & 121.09 & 118.88 & 123.41 & 124.66 \\
\hline TS12 & 68.71 & 75.59 & 70.69 & 71.22 & 72.50 & 73.00 \\
\hline TS13 & 80.09 & 90.64 & 92.99 & 92.23 & 92.90 & 93.86 \\
\hline TS14 & 97.66 & 103.29 & 106.34 & 105.29 & 105.98 & 106.83 \\
\hline TS15 & 91.75 & 98.43 & 95.28 & 94.40 & 95.30 & 96.53 \\
\hline TS16 & 118.74 & 127.97 & 123.64 & 122.48 & 124.23 & 125.45 \\
\hline TS17 & 92.95 & 110.82 & 105.50 & 96.15 & 97.44 & 98.63 \\
\hline TS18 & 116.02 & 121.77 & 117.76 & 117.35 & 118.14 & 119.09 \\
\hline TS19 & 125.22 & 134.09 & 132.95 & 131.68 & 132.80 & - \\
\hline TS20 & 87.30 & 91.86 & 93.86 & 92.85 & 91.00 & 92.14 \\
\hline $\mathrm{TS} 21$ & 120.79 & 128.01 & 129.45 & 128.34 & 128.56 & 129.77 \\
\hline TS22 & 106.84 & 110.91 & 115.21 & 114.10 & 113.24 & 114.31 \\
\hline TS23 & 116.30 & 131.65 & 129.80 & 128.99 & 130.52 & 131.62 \\
\hline TS24 & 119.45 & 124.90 & 127.77 & 126.82 & 126.31 & 126.67 \\
\hline TS25 & 96.33 & 108.19 & 35.02 & 40.95 & 71.00 & 101.72 \\
\hline TS26 & 114.94 & 124.36 & 118.58 & 117.90 & 118.96 & 120.13 \\
\hline TS27 & 98.34 & 110.82 & 105.50 & 103.94 & 105.26 & 106.79 \\
\hline P1 & 65.21 & 72.84 & 73.17 & 72.05 & 73.57 & 74.02 \\
\hline
\end{tabular}




\begin{tabular}{ccccccc} 
P2 & 79.78 & 85.31 & 82.96 & 81.52 & 82.81 & 83.80 \\
P6 & 111.20 & 124.01 & 122.79 & 122.01 & 124.20 & 124.08 \\
P5 & 77.57 & 85.41 & 83.84 & 82.54 & 83.91 & 84.84 \\
P8 & 67.02 & 75.45 & 76.02 & 75.64 & 74.68 & 75.43 \\
P4 & 97.87 & 107.64 & 108.53 & 106.92 & 106.82 & 107.73 \\
P3 & 83.02 & 87.60 & 88.05 & 87.08 & 87.52 & 88.00 \\
P7 & 140.74 & 150.03 & 146.29 & 144.44 & 145.55 & 147.09 \\
\hline
\end{tabular}

Table S2 The Gibbs free energy (1500K) of thiophene intermediates and transition states at different levels. (Unit: $\mathrm{kcal} / \mathrm{mol})$

\begin{tabular}{|c|c|c|c|c|c|c|}
\hline & $\begin{array}{l}\text { B3LYP/ } \\
\text { cc-pVTZ }\end{array}$ & $\begin{array}{c}\text { M062x/ } \\
\text { def2-qzvp }\end{array}$ & $\begin{array}{l}\text { CBS- } \\
\text { QB33 }\end{array}$ & G4 & $\begin{array}{c}\mathrm{CCSD}(\mathrm{T}) \\
/ \mathrm{CBS}\end{array}$ & W1BD \\
\hline $\mathrm{C}_{2,4}$ & 71.94 & 73.29 & 72.09 & 70.62 & 71.31 & 71.60 \\
\hline $\mathrm{C}_{2,5}$ & 51.92 & 53.52 & 53.34 & 52.17 & 52.18 & 52.62 \\
\hline TS- $\mathrm{C}_{2,3}-\mathrm{C}_{2,4}$ & 87.75 & 87.01 & 86.70 & 86.73 & 86.81 & 87.17 \\
\hline TS- $\mathrm{C}_{2,4}-\mathrm{C}_{2,5}$ & 88.18 & 87.46 & 88.53 & 87.69 & 87.59 & 88.06 \\
\hline TS- $\mathrm{C}_{2,5}-\mathrm{C}_{2,1}$ & 93.89 & 94.40 & 93.70 & 93.10 & 92.99 & 93.25 \\
\hline IM1 & 27.30 & 32.56 & 34.44 & 34.27 & 34.65 & 35.26 \\
\hline IM2 & 37.33 & 39.49 & 39.30 & 38.42 & 39.20 & 39.83 \\
\hline IM3 & 32.31 & 34.17 & 33.91 & 33.53 & 33.58 & 33.93 \\
\hline IM4 & 36.51 & 39.15 & 39.09 & 38.15 & 39.27 & 39.53 \\
\hline IM5 & 40.27 & 38.78 & 41.84 & 40.85 & 41.44 & 41.89 \\
\hline IM6 & 17.60 & 20.04 & 24.10 & 23.66 & 24.85 & 25.31 \\
\hline IM7 & 79.89 & 88.16 & 79.24 & 78.77 & 81.05 & 82.01 \\
\hline IM8 & 62.06 & 39.54 & 67.73 & 65.82 & 67.50 & 68.87 \\
\hline IM9 & 45.62 & 47.30 & 49.68 & 49.31 & 49.70 & 50.08 \\
\hline IM10 & 27.33 & 34.10 & 32.02 & 31.34 & 31.22 & 31.76 \\
\hline IM11 & 77.84 & 91.62 & 88.42 & 89.22 & 86.02 & 87.23 \\
\hline BIM6 & 61.79 & 69.21 & 72.20 & 70.67 & 72.07 & 72.46 \\
\hline BIM7 & 54.88 & 84.25 & 66.51 & 87.13 & 68.13 & 68.11 \\
\hline TS1 & 85.94 & 92.70 & 88.86 & 88.83 & 90.59 & 91.27 \\
\hline TS2 & 129.49 & 137.44 & 132.55 & 132.48 & 132.60 & 133.79 \\
\hline TS3 & 92.67 & 92.24 & 91.34 & 91.02 & 91.49 & 92.22 \\
\hline TS4 & 56.48 & 64.18 & 62.53 & 62.57 & 63.39 & 64.26 \\
\hline TS5 & 80.18 & 80.65 & 82.28 & 80.75 & 81.47 & 82.67 \\
\hline TS6 & 92.99 & 95.63 & 94.01 & 93.62 & 94.36 & 94.91 \\
\hline TS7 & 101.73 & 111.79 & 109.23 & 107.98 & 110.75 & 112.02 \\
\hline TS8 & 108.53 & 116.30 & 109.01 & 108.81 & 112.06 & 114.41 \\
\hline TS9 & 96.38 & 102.25 & 99.31 & 99.00 & 100.04 & 101.27 \\
\hline TS 10 & 97.25 & 111.28 & 103.28 & 102.75 & 103.55 & 104.28 \\
\hline TS11 & 113.14 & 127.50 & 109.57 & 107.58 & 111.86 & 113.05 \\
\hline TS12 & 68.26 & 74.83 & 70.31 & 70.74 & 72.04 & 72.54 \\
\hline TS13 & 66.18 & 79.99 & 81.55 & 79.55 & 79.00 & 78.95 \\
\hline TS14 & 87.88 & 92.58 & 96.48 & 95.69 & 96.20 & 97.01 \\
\hline TS 15 & 79.07 & 85.80 & 82.80 & 81.41 & 82.63 & 83.80 \\
\hline TS16 & 107.47 & 117.91 & 112.05 & 111.42 & 112.96 & 114.17 \\
\hline TS17 & 83.58 & 108.28 & 96.24 & 86.66 & 88.07 & 89.20 \\
\hline TS18 & 100.50 & 108.50 & 102.47 & 101.20 & 102.62 & 103.63 \\
\hline TS19 & 58.62 & 68.27 & 66.63 & 64.26 & 66.19 & \\
\hline TS20 & 74.03 & 80.43 & 81.52 & 79.02 & 77.74 & 78.99 \\
\hline TS21 & 97.84 & 101.75 & 106.06 & 105.48 & 105.62 & 106.91 \\
\hline TS22 & 93.06 & 101.83 & 101.56 & 100.15 & 99.46 & 100.51 \\
\hline TS23 & 94.36 & 112.52 & 107.90 & 106.47 & 108.58 & 109.61 \\
\hline TS24 & 105.73 & 110.90 & 113.91 & 110.72 & 112.58 & 113.27 \\
\hline TS25 & 84.53 & 96.24 & 23.66 & 28.93 & 59.21 & 89.88 \\
\hline TS26 & 107.39 & 115.60 & 111.21 & 110.12 & 111.41 & 112.49 \\
\hline TS27 & 89.20 & 108.28 & 96.24 & 94.87 & 96.13 & \\
\hline P1 & 0.45 & 8.21 & 8.52 & 6.95 & 8.81 & 9.38 \\
\hline P2 & 15.98 & 21.44 & 19.31 & 17.50 & 19.01 & 20.12 \\
\hline P6 & 35.58 & 48.47 & 47.35 & 45.87 & 48.57 & 48.59 \\
\hline P5 & 14.52 & 21.86 & 20.95 & 19.77 & 20.87 & 21.88 \\
\hline P8 & 19.44 & 26.95 & 27.53 & 25.83 & 27.10 & 27.97 \\
\hline P4 & 42.23 & 51.78 & 52.98 & 51.17 & 51.17 & 52.11 \\
\hline P3 & 15.46 & 20.55 & 20.65 & 18.78 & 19.96 & 20.58 \\
\hline P7 & 80.82 & 89.70 & 86.59 & 84.61 & 85.63 & 87.32 \\
\hline
\end{tabular}


Table S3 The potential energy (0K) of furan intermediates and transition states at different levels. (Unit: Kcal/mol)

\begin{tabular}{|c|c|c|c|c|c|c|c|}
\hline & $\begin{array}{l}\text { B3LYP/ } \\
\text { cc-pVTZ }\end{array}$ & $\begin{array}{c}\text { M062x/ } \\
\text { def2-qzvp }\end{array}$ & $\begin{array}{l}\text { CBS- } \\
\text { QB3 }\end{array}$ & G4 & $\begin{array}{l}\text { CBS- } \\
\text { APNO }\end{array}$ & $\begin{array}{c}\mathrm{CCSD}(\mathrm{T}) \\
/ \mathrm{CBS}\end{array}$ & W1BD \\
\hline$F-\mathrm{C}_{2,4}$ & 73.25 & 77.09 & 74.88 & 73.92 & 75.33 & 73.79 & 74.83 \\
\hline$F-\mathrm{C}_{2,5}$ & 53.24 & 54.94 & 53.47 & 52.70 & 53.87 & 52.60 & 53.35 \\
\hline$F$-TS-C ${ }_{2,3}-\mathrm{C}_{2,4}$ & 91.78 & 92.23 & 92.21 & 91.91 & 91.65 & 91.77 & 92.48 \\
\hline$F-\mathrm{TS}-\mathrm{C}_{2,4}-\mathrm{C}_{2,5}$ & 90.16 & 90.20 & 91.35 & 90.49 & 90.48 & 90.07 & 90.86 \\
\hline$F-\mathrm{TS}-\mathrm{C}_{2,5}-\mathrm{C}_{2,1}$ & 108.42 & 111.29 & 110.64 & 109.91 & 110.42 & 109.95 & 110.67 \\
\hline$F$-IM1 & 22.30 & 28.15 & 27.51 & 26.86 & 28.28 & 26.29 & 27.99 \\
\hline$F$-IM2 & 30.34 & 32.70 & 31.17 & 30.29 & 31.72 & 30.86 & 31.32 \\
\hline$F$-IM3 & 40.76 & 42.29 & 41.09 & 40.53 & 41.85 & 40.17 & 41.51 \\
\hline$F$-IM4 & 53.66 & 54.27 & 56.41 & 55.98 & 57.04 & 56.41 & 56.50 \\
\hline$F$-IM5 & 54.97 & 53.38 & 54.21 & 53.66 & 54.88 & 54.48 & 54.89 \\
\hline$F$-IM6 & 10.37 & 15.04 & 15.71 & 14.91 & 15.58 & 15.52 & 15.75 \\
\hline$F$-IM9 & 77.33 & 80.85 & 82.35 & 80.06 & 80.69 & 81.89 & 82.36 \\
\hline$F$-IM10 & 30.23 & 31.51 & 32.47 & 31.37 & 32.60 & 31.59 & 31.68 \\
\hline$F$-IM11 & 94.37 & 99.58 & 100.73 & 99.42 & 101.24 & 99.20 & 100.00 \\
\hline$F$-BIM6 & 93.35 & 102.02 & 102.73 & 101.32 & & 102.75 & 102.95 \\
\hline$F$-BIM7 & 66.40 & 74.28 & 74.67 & 74.27 & 75.26 & 75.92 & 75.98 \\
\hline$F$-TS1 & 82.31 & 91.61 & 84.32 & 84.11 & 84.64 & 85.16 & 85.83 \\
\hline$F$-TS2 & 101.62 & 110.66 & 101.54 & 100.86 & 98.38 & 100.78 & 102.28 \\
\hline$F$-TS3 & 96.64 & 97.40 & 95.64 & 95.16 & 95.84 & 95.97 & 96.69 \\
\hline$F$-TS4 & 61.52 & 69.10 & 66.73 & 66.15 & 66.38 & 66.85 & 67.49 \\
\hline$F$-TS5 & 74.84 & 75.86 & 76.17 & 74.67 & 76.06 & 74.93 & 75.94 \\
\hline$F$-TS6 & 101.13 & 105.44 & 103.24 & 102.43 & 102.32 & 103.02 & 103.92 \\
\hline$F$-TS7 & 119.19 & 128.28 & 130.84 & 129.31 & 130.26 & 131.07 & 132.03 \\
\hline$F$-TS8 & 120.29 & 127.55 & 123.10 & 126.53 & 127.86 & 127.22 & 128.15 \\
\hline$F$-TS9 & 68.12 & 73.54 & 71.33 & 71.08 & 70.77 & 71.12 & 72.17 \\
\hline$F$-TS10 & 98.25 & 106.88 & 105.50 & 104.15 & 105.23 & 104.64 & 105.27 \\
\hline$F$-TS11 & 99.17 & 113.24 & 95.68 & 93.26 & 97.23 & 97.12 & 98.08 \\
\hline$F$-TS12 & 65.07 & 75.46 & 66.72 & 66.04 & 65.76 & 66.94 & 67.83 \\
\hline$F$-TS13 & 77.91 & 88.90 & 85.17 & 83.55 & 81.11 & 84.84 & 85.68 \\
\hline$F$-TS14 & 76.06 & 80.41 & 83.35 & 82.10 & 82.46 & 82.12 & 82.76 \\
\hline$F$-TS16 & - & 106.68 & - & - & 104.34 & 104.62 & - \\
\hline$F$-TS18 & 113.40 & 120.92 & 113.65 & 111.79 & 113.64 & 113.25 & 114.13 \\
\hline$F$-TS19 & 137.41 & 144.98 & 142.68 & 141.61 & - & 141.89 & 142.33 \\
\hline$F$-TS20 & 79.79 & 76.19 & 78.98 & 77.41 & 81.70 & 77.19 & 77.84 \\
\hline$F$-TS22 & 96.49 & 99.99 & 101.59 & 100.18 & 101.60 & 99.97 & 100.67 \\
\hline$F$-TS23 & 126.22 & 138.46 & 136.13 & 134.08 & 135.31 & 135.64 & 136.20 \\
\hline$F$-TS24 & 106.66 & 110.72 & 112.00 & 110.62 & 112.14 & 110.44 & 110.83 \\
\hline$F$-TS25 & 105.95 & 119.51 & 91.19 & 89.70 & 101.46 & 113.88 & 110.08 \\
\hline$F$-TS26 & 97.19 & 106.01 & 104.30 & 102.33 & 104.49 & 103.62 & 104.46 \\
\hline$F$-TS27 & 65.48 & 72.08 & 67.77 & 67.66 & 67.09 & 68.04 & 69.08 \\
\hline$F$-P1 & 43.89 & 50.17 & 50.08 & 48.81 & 50.80 & 49.66 & 49.90 \\
\hline$F$-P2 & 24.16 & 26.46 & 24.68 & 22.89 & 24.82 & 23.95 & 24.73 \\
\hline$F$-P6 & 90.07 & 102.03 & 100.20 & 98.17 & 99.85 & 100.25 & 100.69 \\
\hline$F$-P5 & 21.95 & 26.55 & 25.56 & 23.90 & 26.00 & 25.06 & 25.77 \\
\hline$F$-P8 & 57.42 & 60.91 & 58.85 & 57.37 & 58.98 & 57.81 & 57.72 \\
\hline$F-\mathrm{P} 4$ & 131.80 & 137.62 & 136.73 & 134.74 & 136.38 & 135.47 & 135.88 \\
\hline$F$-P3 & 81.06 & 83.37 & 83.87 & 82.59 & 85.35 & 83.24 & 83.28 \\
\hline$F$-P7 & 72.79 & 78.52 & 78.41 & 76.65 & 78.64 & 77.79 & 78.61 \\
\hline
\end{tabular}

Table S4 The Gibbs free energy $(1500 \mathrm{~K})$ of furan intermediates and transition states at different levels. (Unit: Kcal/mol)

\begin{tabular}{cccccccc}
\hline & $\begin{array}{c}\text { B3LYP/ } \\
\text { cc-pVTZ }\end{array}$ & $\begin{array}{c}\text { M062x/ } \\
\text { def2qzvp }\end{array}$ & $\begin{array}{c}\text { CBS- } \\
\text { QB3 }\end{array}$ & G4 & $\begin{array}{c}\text { CBS- } \\
\text { APNO }\end{array}$ & $\begin{array}{c}\text { CCSD(T) } \\
\text { /CBS }\end{array}$ & W1BD \\
\hline$F-C_{2,4}$ & 63.53 & 64.59 & 64.81 & 64.74 & 62.05 & 64.07 & 65.07 \\
$F-C_{2,5}$ & 51.28 & 52.87 & 51.44 & 50.82 & 51.94 & 50.64 & 51.37 \\
$F-\mathrm{TS}_{2,3^{-}}$ & 91.80 & 92.25 & 92.17 & 92.02 & 91.55 & 91.79 & 92.52 \\
$\mathrm{C}_{2,4}$ & & & & & & &
\end{tabular}




\begin{tabular}{|c|c|c|c|c|c|c|c|}
\hline $\begin{array}{c}F-\mathrm{TS}^{-\mathrm{C}_{2,4^{-}}} \\
\mathrm{C}_{2,5}\end{array}$ & 90.53 & 90.66 & 91.65 & 90.96 & 90.87 & 90.44 & 91.25 \\
\hline $\begin{array}{c}F-\mathrm{TS}^{-\mathrm{C}_{2,5^{-}}} \\
\mathrm{C}_{2,1}\end{array}$ & 104.99 & 109.76 & 106.61 & 107.33 & 108.85 & 106.52 & 107.24 \\
\hline$F$-IM1 & 9.12 & 14.55 & 14.58 & 14.13 & 15.01 & 8.06 & 14.78 \\
\hline$F$-IM2 & 16.25 & 18.50 & 17.12 & 15.99 & 18.17 & 16.77 & 17.21 \\
\hline$F$-IM3 & 35.18 & 36.84 & 35.46 & 35.11 & 36.66 & 26.99 & 35.92 \\
\hline$F$-IM4 & 40.57 & 41.29 & 43.52 & 42.77 & 44.84 & 43.32 & 43.37 \\
\hline$F$-IM5 & 45.92 & 44.69 & 45.02 & 44.43 & 46.58 & 45.43 & 45.81 \\
\hline$F$-IM6 & -3.90 & 8.09 & 1.17 & 0.77 & 9.03 & 1.26 & 1.45 \\
\hline$F$-IM9 & 59.38 & 67.62 & 61.42 & 62.69 & 67.13 & 63.94 & 64.32 \\
\hline$F$-IM10 & 17.86 & 19.45 & 20.09 & 18.81 & 20.65 & 19.22 & 19.29 \\
\hline$F$-IM11 & 73.00 & 80.52 & 80.07 & 79.28 & 82.99 & 77.83 & 78.57 \\
\hline$F$-BIM6 & 71.92 & 80.81 & 81.38 & 79.44 & - & 81.32 & 81.46 \\
\hline$F$-BIM7 & 48.48 & 56.25 & 56.70 & 56.27 & 55.70 & 58.00 & 58.01 \\
\hline$F$-TS1 & 70.23 & 80.77 & 72.44 & 71.84 & 74.66 & 73.08 & 73.72 \\
\hline$F$-TS2 & 78.26 & 91.96 & 78.90 & 80.69 & 57.10 & 77.43 & 78.87 \\
\hline$F$-TS3 & 95.69 & 96.55 & 94.77 & 94.30 & 94.89 & 95.02 & 95.75 \\
\hline$F$-TS4 & 56.04 & 63.52 & 61.26 & 60.84 & 60.47 & 61.37 & 62.01 \\
\hline$F$-TS5 & 59.22 & 66.50 & 60.76 & 58.96 & 59.28 & 59.32 & 60.31 \\
\hline$F$-TS6 & 98.30 & 102.30 & 100.42 & 99.70 & 98.37 & 100.19 & 101.09 \\
\hline$F$-TS7 & 94.62 & 109.19 & 106.05 & 101.87 & 109.35 & 106.50 & 107.39 \\
\hline$F$-TS8 & 106.82 & 113.79 & 109.74 & 112.69 & 112.65 & 113.75 & 114.65 \\
\hline$F$-TS9 & 51.14 & 59.45 & 55.11 & 55.37 & 57.03 & 54.15 & 55.16 \\
\hline$F$-TS10 & 74.74 & 86.86 & 82.63 & 81.10 & 82.15 & 81.13 & 81.72 \\
\hline$F$-TS11 & 86.07 & 98.82 & 82.29 & 80.38 & 83.54 & 84.02 & 84.95 \\
\hline$F$-TS12 & 63.07 & 73.31 & 64.67 & 64.14 & 63.16 & 64.94 & 65.83 \\
\hline$F$-TS13 & 65.97 & 77.31 & 73.70 & 71.04 & 68.64 & 72.89 & 73.72 \\
\hline$F$-TS14 & 65.59 & 69.70 & 72.85 & 71.82 & 71.35 & 71.65 & 72.28 \\
\hline$F$-TS16 & - & 92.66 & - & - & 87.48 & 91.40 & - \\
\hline$F$-TS18 & 96.37 & 106.44 & 96.92 & 94.92 & 97.74 & 96.21 & 97.05 \\
\hline$F$-TS19 & 69.14 & 78.36 & 74.86 & 71.35 & - & 73.62 & 74.19 \\
\hline$F$-TS20 & 68.28 & 65.83 & 67.33 & 66.30 & 71.85 & 65.04 & 65.67 \\
\hline$F$-TS22 & 83.90 & 87.84 & 89.10 & 87.73 & 90.49 & 87.38 & 88.06 \\
\hline$F$-TS23 & 106.35 & 120.94 & 116.57 & 114.52 & 119.45 & 115.76 & 116.28 \\
\hline$F$-TS24 & 93.03 & 97.81 & 98.47 & 96.36 & 100.62 & 96.80 & 97.16 \\
\hline$F$-TS25 & 95.23 & 108.37 & 80.13 & 78.65 & 89.53 & 103.16 & 99.35 \\
\hline$F$-TS26 & 84.52 & 93.93 & 91.62 & 89.27 & 93.19 & 90.96 & 91.78 \\
\hline$F$-TS27 & 54.24 & 59.02 & 56.71 & 56.98 & 53.65 & 56.80 & 57.82 \\
\hline$F-\mathrm{P} 1$ & -21.75 & -15.27 & -15.36 & -17.18 & -11.79 & -15.98 & -15.62 \\
\hline$F$-P2 & -40.08 & -37.81 & -39.46 & -41.53 & -38.99 & -40.28 & -39.37 \\
\hline$F$-P6 & 13.76 & 25.89 & 24.01 & 21.40 & 22.61 & 23.95 & 24.53 \\
\hline$F$-P5 & -41.54 & -37.40 & -37.82 & -39.25 & -37.54 & -38.43 & -37.60 \\
\hline$F$-P8 & 10.98 & 13.54 & 11.41 & 8.74 & 16.95 & 11.37 & 11.42 \\
\hline$F$-P4 & 73.60 & 79.28 & 78.55 & 76.47 & 78.47 & 77.27 & 77.74 \\
\hline$F$-P3 & 14.60 & 17.67 & 17.63 & 15.34 & 22.77 & 16.78 & 16.94 \\
\hline$F$-P7 & 4.53 & 10.14 & 10.21 & 8.50 & 9.30 & 9.53 & 10.48 \\
\hline
\end{tabular}

\section{Bond rotation}

Due to the bond rotation generally have low barrier, it is insignificant kinetically and thermodynamically to the reaction. We have simplified some rotation process in the PES figures of manuscript. Here are the detailed information. 


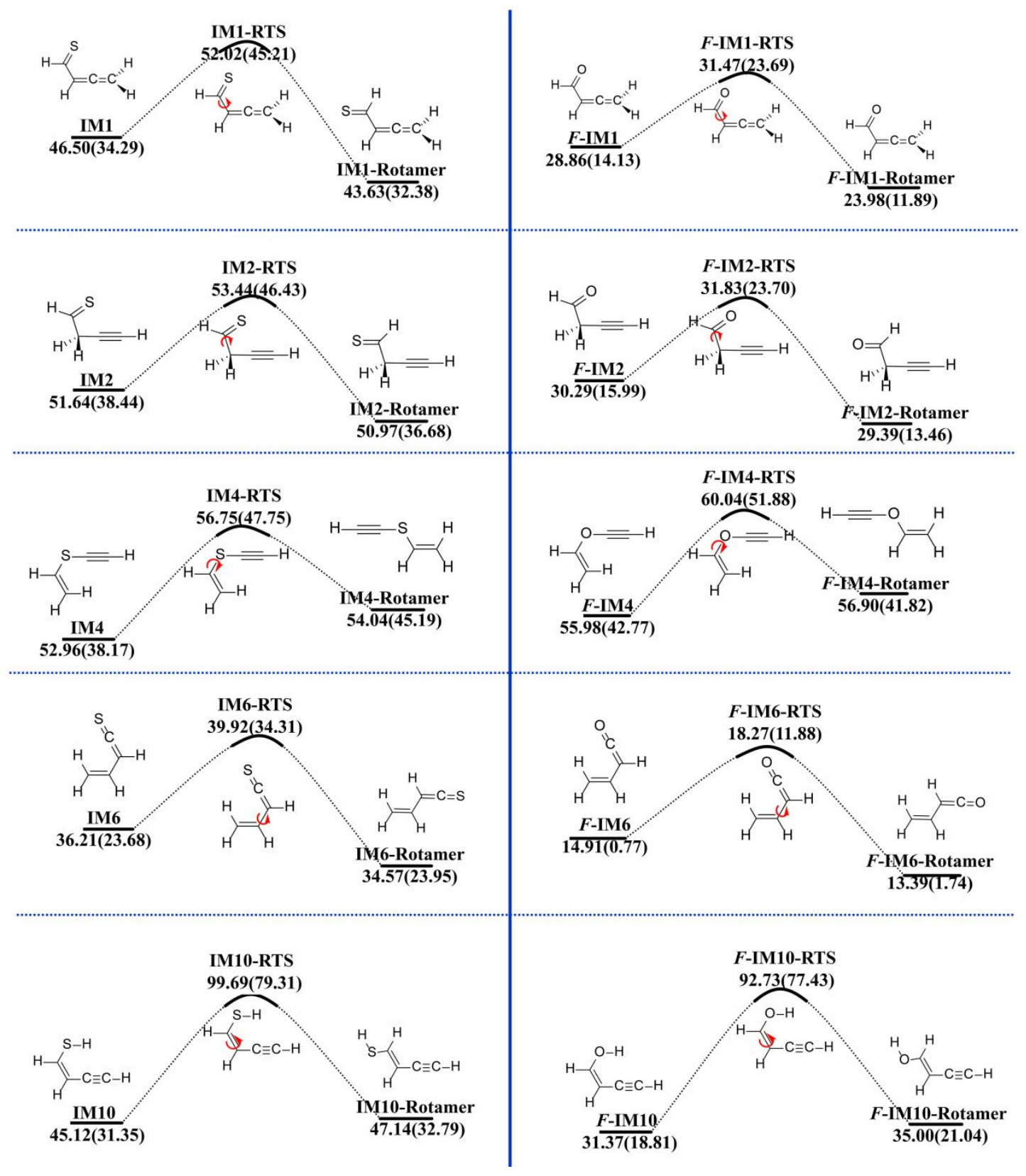

Fig. S1 Potential energy surface (G4 level) of the bond rotation process. The value in bracket are the Gibbs free energy at $1500 \mathrm{~K}$.

\section{Geometries}

Here, the geometries (xyz) optimized at B3LYP/cc-pVTZ level were provided. Readers can use this as an initial structure to obtain The geometries can be easily optimized using this geometry as an initial input. 


\subsection{Geometries in Section 3.1}

Table S1 Geometries appeared in Section 3.1. (unit: $\AA$ )

\begin{tabular}{|c|c|c|c|c|c|c|c|c|c|c|}
\hline & \multicolumn{5}{|c|}{ Thiophene } & \multicolumn{5}{|c|}{ Furan } \\
\hline & 0 & 1 & & & & 0 & 1 & & & \\
\hline \multirow[t]{9}{*}{ Reactant } & & $\mathrm{C}$ & -3.4428 & 1.45324 & $6 \mathrm{E}-06$ & & $\mathrm{C}$ & -3.3075 & 1.50613 & $-3 \mathrm{E}-06$ \\
\hline & & $\mathrm{C}$ & -2.554 & 0.41997 & $5 \mathrm{E}-06$ & & $\mathrm{C}$ & -2.5471 & 0.38513 & 0.0001 \\
\hline & & $\mathrm{C}$ & -1.2002 & 0.85975 & 0.00031 & & $\mathrm{C}$ & -1.1852 & 0.82753 & 0.00025 \\
\hline & & $\mathrm{C}$ & -1.0883 & 2.21813 & $9.3 \mathrm{E}-05$ & & $\mathrm{C}$ & -1.2289 & 2.18138 & $6.7 \mathrm{E}-05$ \\
\hline & & $\mathrm{S}$ & -2.637 & 2.97919 & $-5 \mathrm{E}-05$ & & $\mathrm{O}$ & -2.519 & 2.6158 & $-1 \mathrm{E}-05$ \\
\hline & & $\mathrm{H}$ & -4.5186 & 1.40986 & $-6 \mathrm{E}-05$ & & $\mathrm{H}$ & -4.3677 & 1.68202 & $-9 E-05$ \\
\hline & & $\mathrm{H}$ & -2.8505 & -0.6183 & $-2 \mathrm{E}-05$ & & $\mathrm{H}$ & -2.9044 & -0.6299 & 0.00013 \\
\hline & & $\mathrm{H}$ & -0.35 & 0.19397 & 0.0005 & & $\mathrm{H}$ & -0.2997 & 0.21618 & 0.0004 \\
\hline & & $\mathrm{H}$ & -0.1924 & 2.81523 & 0.00011 & & $\mathrm{H}$ & -0.4744 & 2.94674 & $4.4 \mathrm{E}-05$ \\
\hline \multirow[t]{10}{*}{$\mathrm{C}_{2,3}$} & 0 & 1 & & & & 0 & 1 & & & \\
\hline & & $\mathrm{C}$ & -3.438 & 1.36715 & -0.047 & & $\mathrm{C}$ & -3.3277 & 1.39813 & -0.043 \\
\hline & & $\mathrm{C}$ & -2.526 & 0.40042 & -0.0663 & & $\mathrm{C}$ & -2.516 & 0.35779 & -0.068 \\
\hline & & $\mathrm{C}$ & -1.1109 & 0.91049 & -0.0105 & & $\mathrm{C}$ & -1.1202 & 0.90551 & -0.011 \\
\hline & & $\mathrm{C}$ & -1.037 & 2.40644 & 0.05488 & & $\mathrm{C}$ & -1.2772 & 2.40788 & 0.04896 \\
\hline & & $\mathrm{S}$ & -2.6001 & 2.96498 & 0.04107 & & $\mathrm{O}$ & -2.5793 & 2.61389 & 0.02665 \\
\hline & & $\mathrm{H}$ & -4.5132 & 1.28136 & -0.0765 & & $\mathrm{H}$ & -4.3961 & 1.5224 & -0.0631 \\
\hline & & $\mathrm{H}$ & -2.7631 & -0.6536 & -0.1168 & & $\mathrm{H}$ & -2.8067 & -0.6774 & -0.1192 \\
\hline & & $\mathrm{H}$ & -0.5596 & 0.50654 & 0.84879 & & $\mathrm{H}$ & -0.5427 & 0.58087 & 0.86152 \\
\hline & & $\mathrm{H}$ & -0.5181 & 0.57973 & -0.8734 & & $\mathrm{H}$ & -0.5001 & 0.65447 & -0.8786 \\
\hline \multirow[t]{10}{*}{$\mathrm{C}_{2,1}$} & 0 & 1 & & & & 0 & 1 & & & \\
\hline & & $\mathrm{C}$ & -3.4956 & 1.47891 & 0.04277 & & $\mathrm{C}$ & -3.4133 & 1.41834 & 0.18053 \\
\hline & & $\mathrm{C}$ & -2.6316 & 0.44812 & 0.04344 & & $\mathrm{C}$ & -2.5857 & 0.38777 & 0.02623 \\
\hline & & $\mathrm{C}$ & -1.2226 & 0.80789 & -0.06 & & $\mathrm{C}$ & -1.1961 & 0.81153 & -0.0213 \\
\hline & & $\mathrm{C}$ & -0.8479 & 2.11585 & -0.1573 & & $\mathrm{C}$ & -0.8887 & 2.10999 & 0.07619 \\
\hline & & $\mathrm{S}$ & -2.5001 & 2.92592 & -0.2648 & & $\mathrm{O}$ & -2.7094 & 2.62544 & 0.14467 \\
\hline & & $\mathrm{H}$ & -4.5717 & 1.48594 & 0.05007 & & $\mathrm{H}$ & -4.4878 & 1.46695 & 0.24154 \\
\hline & & $\mathrm{H}$ & -2.9661 & -0.5793 & 0.12394 & & $\mathrm{H}$ & -2.9132 & -0.6362 & -0.0627 \\
\hline & & $\mathrm{H}$ & -0.4643 & 0.03483 & -0.0176 & & $\mathrm{H}$ & -0.3731 & 0.11379 & -0.1141 \\
\hline & & $\mathrm{H}$ & -2.5008 & 3.50319 & 0.95505 & & $\mathrm{H}$ & -2.8226 & 3.18006 & 0.92869 \\
\hline \multirow[t]{10}{*}{ IM1 } & & 0 & 1 & & & 0 & 1 & & & \\
\hline & & $\mathrm{C}$ & -4.1445 & 1.24454 & 0.01127 & & $\mathrm{C}$ & -1.4214 & 0.24089 & 9E-06 \\
\hline & & $\mathrm{C}$ & -2.8632 & 0.55714 & -0.0061 & & $\mathrm{C}$ & -0.1004 & 0.914 & -0.0001 \\
\hline & & $\mathrm{C}$ & -1.6856 & 1.14349 & 0.03807 & & $\mathrm{C}$ & 2.14512 & -0.4044 & 0.00001 \\
\hline & & $\mathrm{C}$ & -0.5268 & 1.71342 & 0.08125 & & $\mathrm{C}$ & 1.03496 & 0.26023 & $-7 E-05$ \\
\hline & & $\mathrm{S}$ & -4.4262 & 2.84943 & 0.086 & & $\mathrm{O}$ & -1.6023 & -0.9518 & 0.00017 \\
\hline & & $\mathrm{H}$ & -4.9958 & 0.56698 & -0.0337 & & $\mathrm{H}$ & -2.2777 & 0.94379 & $-5 \mathrm{E}-05$ \\
\hline & & $\mathrm{H}$ & -2.8927 & -0.5273 & -0.0596 & & $\mathrm{H}$ & -0.1012 & 1.99904 & -0.0002 \\
\hline & & $\mathrm{H}$ & -0.0381 & 1.92319 & 1.02598 & & $\mathrm{H}$ & 2.62396 & -0.6964 & -0.9269 \\
\hline & & $\mathrm{H}$ & -0.014 & 2.01459 & -0.8252 & & $\mathrm{H}$ & 2.62402 & -0.6961 & 0.92704 \\
\hline \multirow[t]{10}{*}{ IM2 } & & 0 & 1 & & & 0 & 1 & & & \\
\hline & & $\mathrm{C}$ & -3.8356 & 1.50198 & 0.00903 & & $\mathrm{C}$ & -3.8212 & 1.57739 & 0.00433 \\
\hline & & $\mathrm{C}$ & -2.6099 & 0.62893 & 0.08032 & & $\mathrm{C}$ & -2.6171 & 0.64884 & 0.07878 \\
\hline & & $\mathrm{C}$ & -1.3154 & 1.28853 & 0.05342 & & $\mathrm{C}$ & -1.3187 & 1.29683 & 0.05221 \\
\hline & & $\mathrm{C}$ & -0.2356 & 1.80617 & 0.03286 & & $\mathrm{C}$ & -0.2475 & 1.83196 & 0.03031 \\
\hline & & $\mathrm{S}$ & -3.9109 & 3.1103 & -0.0958 & & $\mathrm{O}$ & -3.7819 & 2.77187 & -0.0724 \\
\hline & & $\mathrm{H}$ & -4.7561 & 0.91805 & 0.03548 & & $\mathrm{H}$ & -4.7876 & 1.0323 & 0.02807 \\
\hline & & $\mathrm{H}$ & -2.68 & -0.0941 & -0.743 & & $\mathrm{H}$ & -2.7164 & -0.065 & -0.7478 \\
\hline & & $\mathrm{H}$ & -2.701 & 0.01777 & 0.98788 & & $\mathrm{H}$ & -2.737 & 0.04762 & 0.98809 \\
\hline & & $\mathrm{H}$ & 0.71472 & 2.27764 & 0.01205 & & $\mathrm{H}$ & 0.69774 & 2.31348 & 0.01063 \\
\hline \multirow[t]{9}{*}{ ITS1 } & 0 & 1 & & & & 0 & 1 & & & \\
\hline & & $\mathrm{C}$ & -3.2846 & 1.56812 & 0.01426 & & $\mathrm{C}$ & -3.5947 & 1.23198 & 0.09449 \\
\hline & & $\mathrm{C}$ & -2.4812 & 0.47419 & 0.05277 & & $\mathrm{C}$ & -2.7843 & 0.16717 & -0.0796 \\
\hline & & $\mathrm{C}$ & -1.097 & 0.72992 & -0.1787 & & $\mathrm{C}$ & -1.3817 & 0.50173 & 0.08371 \\
\hline & & $\mathrm{C}$ & -0.6245 & 1.9884 & -0.472 & & $\mathrm{C}$ & -1.0684 & 1.77408 & 0.23242 \\
\hline & & $\mathrm{S}$ & -2.3518 & 2.97356 & -0.3924 & & $\mathrm{O}$ & -3.1027 & 2.44662 & 0.26036 \\
\hline & & $\mathrm{H}$ & -4.3469 & 1.62209 & 0.18379 & & $\mathrm{H}$ & -4.6769 & 1.17119 & 0.1405 \\
\hline & & $\mathrm{H}$ & -2.8646 & -0.5131 & 0.27941 & & $\mathrm{H}$ & -3.1299 & -0.8388 & -0.2511 \\
\hline & & $\mathrm{H}$ & -0.3705 & -0.0751 & -0.1475 & & $\mathrm{H}$ & -0.6088 & -0.2585 & 0.10889 \\
\hline
\end{tabular}




\begin{tabular}{|c|c|c|c|c|c|c|c|c|c|c|}
\hline & & $\mathrm{H}$ & -1.4099 & 2.96391 & 0.65941 & & $\mathrm{H}$ & -1.9679 & 2.55727 & 0.66714 \\
\hline \multirow[t]{10}{*}{ ITS2 } & 0 & 1 & & & & 0 & 1 & & & \\
\hline & & $\mathrm{C}$ & -3.8524 & 1.12847 & -0.0167 & & $\mathrm{C}$ & -3.6843 & 1.2613 & -0.0148 \\
\hline & & $\mathrm{C}$ & -2.8352 & 0.11236 & 0.09115 & & $\mathrm{C}$ & -2.8247 & 0.11219 & 0.07887 \\
\hline & & $\mathrm{C}$ & -1.4534 & 0.56808 & 0.06092 & & $\mathrm{C}$ & -1.4351 & 0.52987 & 0.05697 \\
\hline & & $\mathrm{C}$ & -1.3818 & 1.91285 & -0.0051 & & $\mathrm{C}$ & -1.4955 & 1.87003 & 0.00524 \\
\hline & & $\mathrm{S}$ & -2.9815 & 2.62298 & 0.00062 & & $\mathrm{O}$ & -2.7836 & 2.31659 & 0.01991 \\
\hline & & $\mathrm{H}$ & -3.4733 & 0.4103 & 1.1125 & & $\mathrm{H}$ & -3.463 & 0.51267 & 1.09954 \\
\hline & & $\mathrm{H}$ & -3.1001 & -0.9327 & -0.0251 & & $\mathrm{H}$ & -3.1961 & -0.8959 & -0.0326 \\
\hline & & $\mathrm{H}$ & -0.599 & -0.0922 & 0.08514 & & $\mathrm{H}$ & -0.5536 & -0.0859 & 0.07744 \\
\hline & & $\mathrm{H}$ & -0.4895 & 2.51812 & -0.0482 & & $\mathrm{H}$ & -0.7302 & 2.62735 & -0.0355 \\
\hline \multirow[t]{10}{*}{ ITS3 } & 0 & 1 & & & & 0 & 1 & & & \\
\hline & & $\mathrm{C}$ & -3.665 & 1.17276 & 0.00164 & & $\mathrm{C}$ & -3.5245 & 1.21959 & 0.0096 \\
\hline & & $\mathrm{C}$ & -2.7841 & 0.13368 & 0.01995 & & $\mathrm{C}$ & -2.7653 & 0.10089 & 0.02262 \\
\hline & & $\mathrm{C}$ & -1.3865 & 0.49204 & -0.0081 & & $\mathrm{C}$ & -1.3549 & 0.46973 & -0.0037 \\
\hline & & $\mathrm{C}$ & -1.323 & 1.89795 & 0.11017 & & $\mathrm{C}$ & -1.4598 & 1.87293 & 0.10177 \\
\hline & & $\mathrm{S}$ & -2.9209 & 2.72679 & 0.06863 & & $\mathrm{O}$ & -2.7756 & 2.34691 & 0.06794 \\
\hline & & $\mathrm{H}$ & -4.7433 & 1.11145 & -0.026 & & $\mathrm{H}$ & -4.5902 & 1.38187 & -0.0155 \\
\hline & & $\mathrm{H}$ & -3.1194 & -0.8941 & 0.01428 & & $\mathrm{H}$ & -3.145 & -0.9075 & 0.00732 \\
\hline & & $\mathrm{H}$ & -1.0623 & 1.22715 & 1.11701 & & $\mathrm{H}$ & -1.1163 & 1.25235 & 1.12027 \\
\hline & & $\mathrm{H}$ & -0.4425 & 2.51043 & -0.0272 & & $\mathrm{H}$ & -0.7152 & 2.64134 & -0.0401 \\
\hline \multirow[t]{10}{*}{ ITS4 } & 0 & 1 & & & & 0 & 1 & & & \\
\hline & & $\mathrm{C}$ & -3.7033 & 1.21259 & 0.03675 & & $\mathrm{C}$ & -3.5591 & 1.26027 & 0.0287 \\
\hline & & $\mathrm{C}$ & -2.7671 & 0.14262 & 0.12946 & & $\mathrm{C}$ & -2.7582 & 0.11076 & 0.13597 \\
\hline & & $\mathrm{C}$ & -1.329 & 0.53708 & 0.04037 & & $\mathrm{C}$ & -1.2885 & 0.53136 & 0.04478 \\
\hline & & $\mathrm{C}$ & -1.3251 & 1.91994 & 0.01096 & & $\mathrm{C}$ & -1.4557 & 1.88978 & 0.01285 \\
\hline & & $\mathrm{S}$ & -2.8995 & 2.67361 & -0.0628 & & $\mathrm{O}$ & -2.7898 & 2.3188 & -0.0313 \\
\hline & & $\mathrm{H}$ & -4.7794 & 1.15758 & 0.00365 & & $\mathrm{H}$ & -4.6231 & 1.42398 & -0.0129 \\
\hline & & $\mathrm{H}$ & -3.0874 & -0.8864 & 0.02636 & & $\mathrm{H}$ & -3.1232 & -0.8992 & 0.02778 \\
\hline & & $\mathrm{H}$ & -2.1868 & 0.19084 & 1.16626 & & $\mathrm{H}$ & -2.1906 & 0.18287 & 1.16539 \\
\hline & & $\mathrm{H}$ & -0.4735 & 2.58077 & -0.0359 & & $\mathrm{H}$ & -0.763 & 2.70998 & -0.0562 \\
\hline \multirow[t]{10}{*}{ BIM1 } & 0 & 3 & & & & 0 & 3 & & & \\
\hline & & $\mathrm{C}$ & -3.6689 & 1.43548 & -0.0 & & $\mathrm{C}$ & -3.6842 & 1.53676 & -0.0029 \\
\hline & & $\mathrm{C}$ & -2.5797 & 0.55696 & $6.5 \mathrm{E}-05$ & & $\mathrm{C}$ & -2.5807 & 0.60028 & $-6 \mathrm{E}-05$ \\
\hline & & $\mathrm{C}$ & -1.2252 & 0.97377 & 0.00036 & & $\mathrm{C}$ & -1.2394 & 0.99917 & 0.00029 \\
\hline & & $\mathrm{C}$ & -0.8687 & 2.26966 & -0.0018 & & $\mathrm{C}$ & -0.8022 & 2.27534 & -0.0019 \\
\hline & & $\mathrm{S}$ & -3.549 & 3.09578 & -0.0057 & & $\mathrm{O}$ & -3.5606 & 2.75034 & -0.0049 \\
\hline & & $\mathrm{H}$ & -4.6598 & 0.99173 & -0.0025 & & $\mathrm{H}$ & -4.6912 & 1.0807 & -0.0026 \\
\hline & & $\mathrm{H}$ & -2.7906 & -0.5063 & 0.00194 & & $\mathrm{H}$ & -2.819 & -0.4552 & 0.00183 \\
\hline & & $\mathrm{H}$ & -0.4682 & 0.18858 & 0.00248 & & $\mathrm{H}$ & -0.4917 & 0.20361 & 0.0025 \\
\hline & & $\mathrm{H}$ & 0.1158 & 2.71844 & -0.0019 & & $\mathrm{H}$ & 0.1747 & 2.73304 & -0.0019 \\
\hline \multirow[t]{10}{*}{ BIM2 } & 0 & 5 & & & & 0 & 5 & & & \\
\hline & & $\mathrm{C}$ & -3.1969 & 1.43951 & -0.0662 & & $\mathrm{C}$ & -3.0037 & 1.54433 & -0.0178 \\
\hline & & $\mathrm{C}$ & -2.5661 & 0.21214 & 0.08695 & & $\mathrm{C}$ & -2.5144 & 0.25761 & 0.05894 \\
\hline & & $\mathrm{C}$ & -1.3395 & -0.1418 & -0.362 & & $\mathrm{C}$ & -1.3231 & -0.2052 & -0.3973 \\
\hline & & $\mathrm{C}$ & -1.0497 & 3.19698 & -0.0669 & & $\mathrm{C}$ & -1.2162 & 3.08728 & -0.0281 \\
\hline & & $\mathrm{S}$ & -2.4932 & 2.85787 & -0.8795 & & $\mathrm{O}$ & -2.3083 & 2.58848 & -0.612 \\
\hline & & $\mathrm{H}$ & -4.2126 & 1.58368 & 0.27356 & & $\mathrm{H}$ & -3.9946 & 1.82182 & 0.31019 \\
\hline & & $\mathrm{H}$ & -3.1375 & -0.546 & 0.63033 & & $\mathrm{H}$ & -3.1902 & -0.4559 & 0.53555 \\
\hline & & $\mathrm{H}$ & -0.7353 & -1.033 & -0.3437 & & $\mathrm{H}$ & -0.8195 & -1.1564 & -0.4181 \\
\hline & & $\mathrm{H}$ & -0.8739 & 3.90891 & 0.73376 & & $\mathrm{H}$ & -1.2348 & 3.99631 & 0.57506 \\
\hline \multirow[t]{10}{*}{ BIM3 } & 0 & 3 & & & & 0 & 3 & & & \\
\hline & & $\mathrm{C}$ & -3.6881 & 1.42427 & -0.0916 & & $\mathrm{C}$ & -3.4434 & 1.43971 & -0.1035 \\
\hline & & $\mathrm{C}$ & -3.4941 & 0.37173 & 0.65171 & & $\mathrm{C}$ & -3.4436 & 0.38808 & 0.67687 \\
\hline & & $\mathrm{C}$ & 0.17875 & 1.82767 & -0.7667 & & $\mathrm{C}$ & -0.0263 & 1.89558 & -0.8187 \\
\hline & & $\mathrm{C}$ & -0.961 & 1.85336 & -0.1375 & & $\mathrm{C}$ & -1.1238 & 1.78268 & -0.1194 \\
\hline & & $\mathrm{S}$ & -2.467 & 2.44582 & -0.879 & & $\mathrm{O}$ & -2.3654 & 2.09784 & -0.6266 \\
\hline & & $\mathrm{H}$ & -4.6904 & 1.79648 & -0.306 & & $\mathrm{H}$ & -4.365 & 1.91308 & -0.4346 \\
\hline & & $\mathrm{H}$ & -4.0582 & -0.3609 & 1.19999 & & $\mathrm{H}$ & -4.1632 & -0.2405 & 1.16572 \\
\hline & & $\mathrm{H}$ & 1.21231 & 1.6032 & -0.5696 & & $\mathrm{H}$ & 1.02897 & 1.78673 & -0.6523 \\
\hline & & $\mathrm{H}$ & -1.0609 & 1.57432 & 0.911 & & $\mathrm{H}$ & -1.1268 & 1.47284 & 0.92481 \\
\hline \multirow[t]{5}{*}{ BIM4 } & 0 & 3 & & & & 0 & 3 & & & \\
\hline & & $\mathrm{C}$ & -3.6562 & 1.47953 & -0.0021 & & $\mathrm{C}$ & -3.6487 & 1.54012 & -0.0024 \\
\hline & & $\mathrm{C}$ & -2.6131 & 0.5588 & 0.00182 & & $\mathrm{C}$ & -2.5656 & 0.58113 & 0.00173 \\
\hline & & $\mathrm{C}$ & -2.7861 & -0.8584 & 0.0071 & & $\mathrm{C}$ & -2.7741 & -0.8025 & 0.00691 \\
\hline & & $\mathrm{C}$ & -3.9418 & -1.522 & 0.0091 & & $\mathrm{C}$ & -3.9642 & -1.4397 & 0.00882 \\
\hline
\end{tabular}




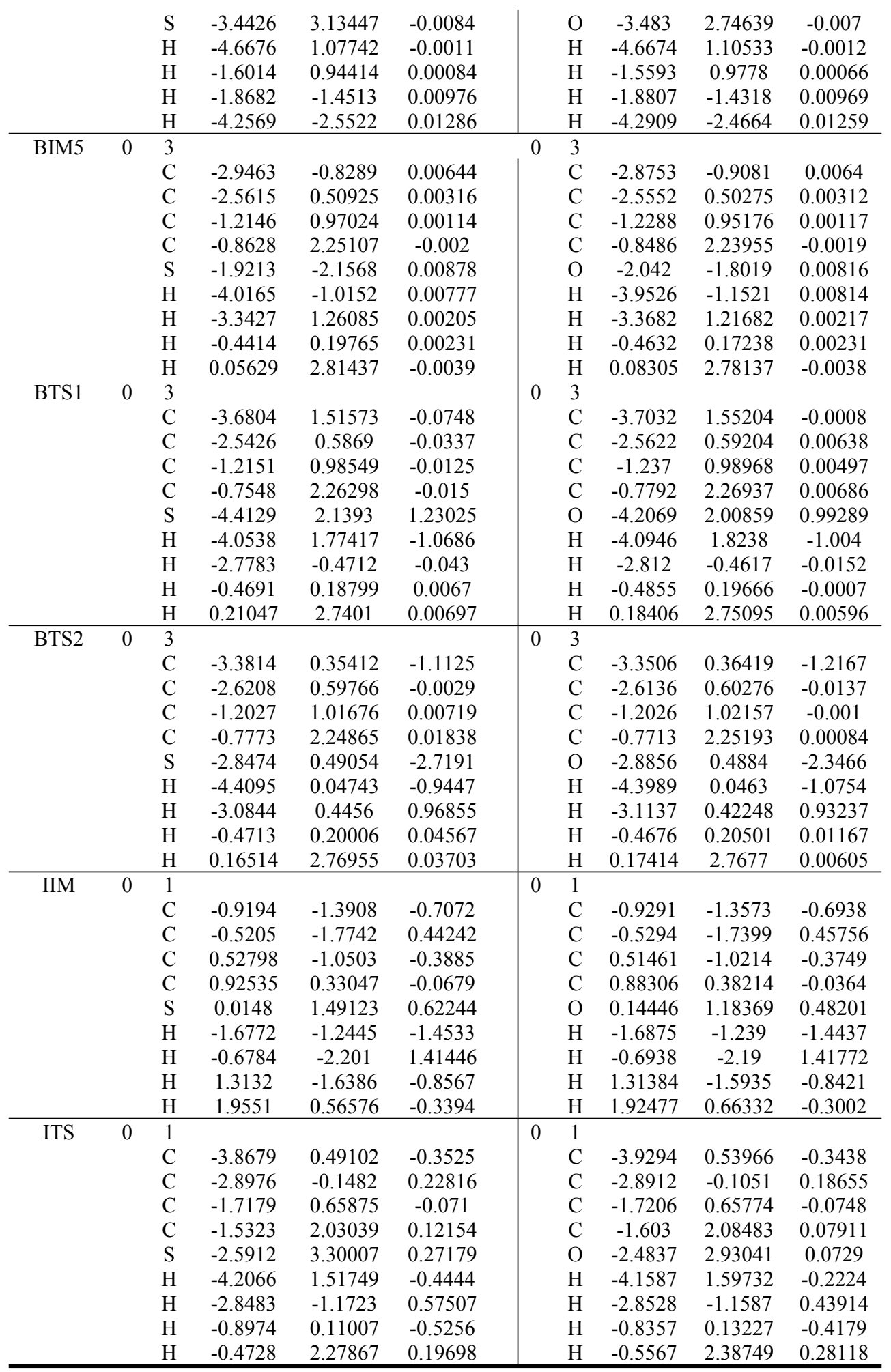

\subsection{Geometries in Section 3.2}

Here, the geometries (xyz) optimized at B3LYP/cc-pVTZ level were provided. Readers can use this as an initial structure to obtain The geometries can be easily optimized using this geometry as an initial input. 
Table S2 Geometries appeared in Section 3.1. (unit: $\AA$ )

\begin{tabular}{|c|c|c|c|c|c|c|c|c|c|c|}
\hline & & & Thio & phene & & & & & & \\
\hline $\mathrm{C}_{2,4}$ & 0 & 1 & & & & 0 & 1 & & & \\
\hline & & $\mathrm{C}$ & 0.11629 & 1.20448 & -0.0003 & & $\mathrm{C}$ & -3.3977 & 1.56044 & 0.04863 \\
\hline & & $\mathrm{C}$ & -1.2607 & 0.67954 & $5.5 \mathrm{E}-05$ & & $\mathrm{C}$ & -2.5616 & 0.32881 & 0.12775 \\
\hline & & $\mathrm{C}$ & -1.1706 & -0.8175 & -0.0004 & & $\mathrm{C}$ & -1.1494 & 0.81579 & 0.06116 \\
\hline & & $\mathrm{C}$ & 0.04523 & -1.4333 & -0.0002 & & $\mathrm{C}$ & -0.8156 & 2.08653 & -0.0447 \\
\hline & & $\mathrm{S}$ & 1.18404 & -0.0502 & 0.00029 & & $\mathrm{O}$ & -2.8509 & 2.63795 & -0.0471 \\
\hline & & $\mathrm{H}$ & 0.38585 & 2.25093 & -0.0001 & & $\mathrm{H}$ & -4.4942 & 1.48844 & 0.07628 \\
\hline & & $\mathrm{H}$ & -1.8101 & 1.07109 & 0.86879 & & $\mathrm{H}$ & -2.8343 & -0.3504 & -0.6897 \\
\hline & & $\mathrm{H}$ & -2.0914 & -1.3902 & -0.0002 & & $\mathrm{H}$ & -0.3125 & 0.12478 & 0.09812 \\
\hline & & $\mathrm{H}$ & -1.8105 & 1.07141 & -0.8683 & & $\mathrm{H}$ & -2.7989 & -0.2148 & 1.05083 \\
\hline $\mathrm{C}_{2,5}$ & 0 & 1 & & & & 0 & 1 & & & \\
\hline & & $\mathrm{C}$ & 0.10839 & 1.27885 & 0.00011 & & $\mathrm{C}$ & -3.4167 & 1.49798 & 0.10688 \\
\hline & & $\mathrm{C}$ & 1.35756 & 0.48328 & $-2 \mathrm{E}-05$ & & $\mathrm{C}$ & -2.5187 & 0.3258 & 0.05614 \\
\hline & & $\mathrm{C}$ & 1.18636 & -0.8555 & -0.0001 & & $\mathrm{C}$ & -1.2571 & 0.76678 & -0.0409 \\
\hline & & $\mathrm{C}$ & -0.1838 & -1.3584 & $-6 \mathrm{E}-05$ & & $\mathrm{C}$ & -1.2244 & 2.2473 & -0.0612 \\
\hline & & $\mathrm{S}$ & -1.198 & -0.0342 & $1.7 \mathrm{E}-05$ & & $\mathrm{O}$ & -2.4863 & 2.62325 & 0.02556 \\
\hline & & $\mathrm{H}$ & 0.00729 & 1.91426 & 0.88313 & & $\mathrm{H}$ & -4.1099 & 1.57106 & -0.7333 \\
\hline & & $\mathrm{H}$ & 2.323 & 0.97396 & $-4 \mathrm{E}-05$ & & $\mathrm{H}$ & -2.87 & -0.6949 & 0.09375 \\
\hline & & $\mathrm{H}$ & 2.01995 & -1.5457 & -0.0002 & & $\mathrm{H}$ & -0.3658 & 0.16239 & -0.0984 \\
\hline & & $\mathrm{H}$ & 0.00725 & 1.91456 & -0.8827 & & $\mathrm{H}$ & -3.9819 & 1.59304 & 1.03599 \\
\hline TS of $\mathrm{C}_{2,3}-\mathrm{C}_{2,4}$ & 0 & 1 & & & & 0 & 1 & & & \\
\hline & & $\mathrm{C}$ & -0.0447 & 1.23761 & -0.0206 & & $\mathrm{C}$ & -3.3085 & 1.52925 & 0.01893 \\
\hline & & $\mathrm{C}$ & 1.23875 & 0.69756 & -0.0415 & & $\mathrm{C}$ & -2.5526 & 0.37291 & 0.08943 \\
\hline & & $\mathrm{C}$ & 1.17334 & -0.8173 & -0.0614 & & $\mathrm{C}$ & -1.1241 & 0.88822 & -0.0107 \\
\hline & & $\mathrm{C}$ & -0.1034 & -1.3741 & -0.0106 & & $\mathrm{C}$ & -1.1337 & 2.27975 & -0.0617 \\
\hline & & $\mathrm{S}$ & -1.1793 & -0.0437 & 0.0032 & & $\mathrm{O}$ & -2.5162 & 2.58726 & -0.0572 \\
\hline & & $\mathrm{H}$ & -0.3011 & 2.2839 & 0.01194 & & $\mathrm{H}$ & -4.3735 & 1.68694 & 0.05225 \\
\hline & & $\mathrm{H}$ & 2.14539 & 1.26717 & -0.1935 & & $\mathrm{H}$ & -2.909 & -0.641 & 0.01036 \\
\hline & & $\mathrm{H}$ & 2.09583 & -1.3823 & -0.0659 & & $\mathrm{H}$ & -0.2596 & 0.24751 & 0.01788 \\
\hline & & $\mathrm{H}$ & 1.34503 & 0.06756 & 1.00018 & & $\mathrm{H}$ & -1.9197 & 0.54226 & 1.10714 \\
\hline $\mathrm{TS}$ of $\mathrm{C}_{2,4}-\mathrm{C}_{2,5}$ & 0 & 1 & & & & 0 & 1 & & & \\
\hline & & $\mathrm{C}$ & -0.0202 & 1.21899 & -0.0541 & & $\mathrm{C}$ & -3.3236 & 1.56931 & 0.01691 \\
\hline & & $\mathrm{C}$ & 1.28514 & 0.59494 & -0.0433 & & $\mathrm{C}$ & -2.5249 & 0.38364 & 0.06461 \\
\hline & & $\mathrm{C}$ & 1.18015 & -0.8347 & -0.018 & & $\mathrm{C}$ & -1.18 & 0.85124 & -0.0066 \\
\hline & & $\mathrm{C}$ & -0.0932 & -1.3844 & 0.0055 & & $\mathrm{C}$ & -1.1246 & 2.23647 & -0.0709 \\
\hline & & $\mathrm{S}$ & -1.1894 & -0.0375 & -0.002 & & $\mathrm{O}$ & -2.5321 & 2.61511 & -0.0364 \\
\hline & & $\mathrm{H}$ & 0.85614 & 1.03018 & 1.03623 & & $\mathrm{H}$ & -4.3966 & 1.68476 & -0.0168 \\
\hline & & $\mathrm{H}$ & 2.1871 & 1.16423 & -0.235 & & $\mathrm{H}$ & -2.9238 & -0.6168 & -0.0252 \\
\hline & & $\mathrm{H}$ & 2.07354 & -1.4447 & 0.01048 & & $\mathrm{H}$ & -0.3118 & 0.21529 & 0.02276 \\
\hline & & $\mathrm{H}$ & -0.1973 & 2.282 & -0.1203 & & $\mathrm{H}$ & -3.0364 & 0.81396 & 1.15015 \\
\hline TS of $\mathrm{C}_{2,5}-\mathrm{C}_{2,1}$ & 0 & 1 & & & & 0 & 1 & & & \\
\hline & & $\mathrm{C}$ & -0.1619 & 1.2836 & -0.0432 & & $\mathrm{C}$ & -3.4187 & 1.40335 & 0.16369 \\
\hline & & $\mathrm{C}$ & -1.3215 & 0.57289 & -0.0113 & & $\mathrm{C}$ & -2.5417 & 0.37424 & 0.03962 \\
\hline & & $\mathrm{C}$ & -1.1624 & -0.8466 & -0.006 & & $\mathrm{C}$ & -1.2056 & 0.83129 & -0.0372 \\
\hline & & $\mathrm{C}$ & 0.11197 & -1.3877 & 0.03155 & & $\mathrm{C}$ & -1.0982 & 2.20389 & 0.09018 \\
\hline & & $\mathrm{S}$ & 1.16856 & -0.0246 & -0.0541 & & $\mathrm{O}$ & -2.4279 & 2.67278 & 0.11944 \\
\hline & & $\mathrm{H}$ & 0.03043 & 2.34133 & -0.109 & & $\mathrm{H}$ & -4.4316 & 1.59875 & -0.1409 \\
\hline & & $\mathrm{H}$ & -2.2881 & 1.05929 & 0.0196 & & $\mathrm{H}$ & -2.8475 & -0.6611 & 0.01883 \\
\hline & & $\mathrm{H}$ & -2.0349 & -1.4893 & 0.01275 & & $\mathrm{H}$ & -0.3534 & 0.17798 & -0.133 \\
\hline & & $\mathrm{H}$ & 0.79826 & 0.7486 & 1.11603 & & $\mathrm{H}$ & -2.9726 & 2.39025 & 1.05998 \\
\hline IM3 & 0 & 1 & & & & 0 & 1 & & & \\
\hline & & $\mathrm{C}$ & -1.2815 & 0.75904 & $8.4 \mathrm{E}-05$ & & $\mathrm{C}$ & 1.41331 & 0.06984 & -0.0001 \\
\hline & & $\mathrm{C}$ & -0.0698 & 1.33763 & $-3 E-05$ & & $\mathrm{C}$ & 0.63593 & -1.0219 & $2.6 \mathrm{E}-05$ \\
\hline & & $\mathrm{C}$ & 2.03743 & -0.1734 & $6.5 \mathrm{E}-05$ & & $\mathrm{C}$ & -1.8179 & -0.0357 & $-9 E-05$ \\
\hline & & $\mathrm{C}$ & 0.74887 & 0.12552 & $2.8 \mathrm{E}-05$ & & $\mathrm{C}$ & -0.5011 & -0.0941 & $-1 \mathrm{E}-05$ \\
\hline & & $\mathrm{S}$ & -0.7321 & -0.9505 & $-7 \mathrm{E}-05$ & & $\mathrm{O}$ & 0.3911 & 1.03028 & 0.0002 \\
\hline & & $\mathrm{H}$ & -2.2955 & 1.12357 & 0.00015 & & $\mathrm{H}$ & 2.45087 & 0.36022 & -0.0002 \\
\hline & & $\mathrm{H}$ & 0.2305 & 2.37466 & $-5 E-05$ & & $\mathrm{H}$ & 0.76784 & -2.0867 & $5 \mathrm{E}-06$ \\
\hline & & $\mathrm{H}$ & 2.3867 & -1.1959 & $6.5 \mathrm{E}-05$ & & $\mathrm{H}$ & -2.3258 & 0.91747 & $-3 \mathrm{E}-05$ \\
\hline & & $\mathrm{H}$ & 2.78217 & 0.61231 & $8.6 \mathrm{E}-05$ & & $\mathrm{H}$ & -2.4032 & -0.9425 & -0.0002 \\
\hline TS1 & 0 & 1 & & & & 0 & 1 & & & \\
\hline & & $\mathrm{C}$ & -1.0728 & -1.3605 & $-1 \mathrm{E}-06$ & & $\mathrm{C}$ & -1.3966 & -0.7668 & -0.0003 \\
\hline
\end{tabular}




\begin{tabular}{|c|c|c|c|c|c|c|c|c|c|c|}
\hline & & $\mathrm{C}$ & -1.7262 & -0.312 & 0 & & $\mathrm{C}$ & -1.2861 & 0.46711 & -0.0001 \\
\hline & & $\mathrm{C}$ & -0.6244 & 1.44227 & $2 \mathrm{E}-06$ & & $\mathrm{C}$ & 0.4697 & 1.19594 & $3.3 \mathrm{E}-05$ \\
\hline & & $\mathrm{C}$ & 0.65987 & 0.99346 & $1 \mathrm{E}-06$ & & $\mathrm{C}$ & 1.25538 & 0.04701 & 0.00019 \\
\hline & & $\mathrm{S}$ & 1.38128 & -0.3836 & $-1 \mathrm{E}-06$ & & $\mathrm{O}$ & 1.02179 & -1.1033 & 0.00021 \\
\hline & & $\mathrm{H}$ & -0.851 & -2.4005 & $-3 \mathrm{E}-06$ & & $\mathrm{H}$ & -1.6782 & -1.7904 & $-3 \mathrm{E}-05$ \\
\hline & & $\mathrm{H}$ & -2.7071 & 0.11774 & $1 \mathrm{E}-06$ & & $\mathrm{H}$ & -1.8812 & 1.36281 & -0.0003 \\
\hline & & $\mathrm{H}$ & -0.9804 & 1.9204 & -0.9087 & & $\mathrm{H}$ & 0.56546 & 1.79701 & -0.9016 \\
\hline & & $\mathrm{H}$ & -0.9804 & 1.9204 & 0.90867 & & $\mathrm{H}$ & 0.56532 & 1.79714 & 0.90159 \\
\hline \multirow[t]{10}{*}{ TS2 } & 0 & 1 & & & & 0 & 1 & & & \\
\hline & & $\mathrm{C}$ & 1.07699 & -1.1286 & $5 \mathrm{E}-06$ & & $\mathrm{C}$ & -1.6739 & 0.37671 & 0.00028 \\
\hline & & $\mathrm{C}$ & -1.1852 & -1.1974 & $5.1 \mathrm{E}-05$ & & $\mathrm{C}$ & 0.47269 & 1.31383 & $-1 \mathrm{E}-05$ \\
\hline & & $\mathrm{C}$ & -1.454 & 0.18828 & -0.0002 & & $\mathrm{C}$ & 1.17041 & 0.0729 & $-8 \mathrm{E}-05$ \\
\hline & & $\mathrm{C}$ & -1.2058 & 1.40673 & $3.7 \mathrm{E}-05$ & & $\mathrm{C}$ & 1.28417 & -1.1857 & -0.0001 \\
\hline & & $\mathrm{S}$ & 1.44909 & 0.37705 & $-1 \mathrm{E}-05$ & & $\mathrm{O}$ & -1.5687 & -0.7553 & 0.00015 \\
\hline & & $\mathrm{H}$ & -1.2448 & -1.7505 & -0.9261 & & $\mathrm{H}$ & 0.43145 & 1.8836 & -0.9199 \\
\hline & & $\mathrm{H}$ & -1.2446 & -1.7501 & 0.92643 & & $\mathrm{H}$ & 0.43123 & 1.88349 & 0.91991 \\
\hline & & $\mathrm{H}$ & -2.4818 & -0.5438 & $2.6 \mathrm{E}-05$ & & $\mathrm{H}$ & 2.10524 & 0.7353 & -0.0012 \\
\hline & & $\mathrm{H}$ & -1.6061 & 2.39735 & 0.00026 & & $\mathrm{H}$ & 2.06121 & -1.9262 & -0.0003 \\
\hline \multirow[t]{10}{*}{ TS3 } & 0 & 1 & & & & 0 & 1 & & & \\
\hline & & $\mathrm{C}$ & 0.56578 & 1.11566 & 0.28655 & & $\mathrm{C}$ & 1.14688 & 0.31674 & 0.28051 \\
\hline & & $\mathrm{C}$ & -0.718 & 0.98232 & -0.1932 & & $\mathrm{C}$ & 0.06261 & 1.02979 & -0.1401 \\
\hline & & $\mathrm{C}$ & -1.5378 & -0.6248 & 0.3389 & & $\mathrm{C}$ & -1.4239 & -0.1493 & 0.31186 \\
\hline & & $\mathrm{C}$ & -0.5881 & -0.4731 & -0.6607 & & $\mathrm{C}$ & -0.4622 & -0.3467 & -0.6569 \\
\hline & & $\mathrm{S}$ & 1.11802 & -0.517 & -0.001 & & $\mathrm{O}$ & 0.72309 & -0.9372 & -0.0445 \\
\hline & & $\mathrm{H}$ & 1.17841 & 1.99875 & 0.3833 & & $\mathrm{H}$ & 2.1785 & 0.52723 & 0.51425 \\
\hline & & $\mathrm{H}$ & -1.4696 & 1.72283 & -0.4152 & & $\mathrm{H}$ & -0.1989 & 2.06208 & -0.2702 \\
\hline & & $\mathrm{H}$ & -1.358 & -1.0669 & 1.31631 & & $\mathrm{H}$ & -1.4169 & -0.65 & 1.27716 \\
\hline & & $\mathrm{H}$ & -2.5706 & -0.3835 & 0.10317 & & $\mathrm{H}$ & -2.2871 & 0.45511 & 0.06216 \\
\hline \multirow[t]{10}{*}{ TS4 } & 0 & 1 & & & & 0 & 1 & & & \\
\hline & & $\mathrm{C}$ & -1.0208 & 0.88693 & 0.13207 & & $\mathrm{C}$ & -1.3781 & 0.03096 & 0.09965 \\
\hline & & $\mathrm{C}$ & 0.28055 & 1.2827 & -0.1152 & & $\mathrm{C}$ & -0.4682 & 1.06084 & -0.0674 \\
\hline & & $\mathrm{C}$ & 2.14074 & -0.5633 & 0.08039 & & $\mathrm{C}$ & 1.88787 & -0.1581 & 0.05956 \\
\hline & & $\mathrm{C}$ & 1.06854 & 0.15254 & -0.1142 & & $\mathrm{C}$ & 0.66052 & 0.25412 & -0.0861 \\
\hline & & $\mathrm{S}$ & -1.1769 & -0.7904 & -0.0215 & & $\mathrm{O}$ & -0.7561 & -1.0828 & -0.0523 \\
\hline & & $\mathrm{H}$ & -1.8362 & 1.53905 & 0.4201 & & $\mathrm{H}$ & -2.4317 & 0.05731 & 0.37414 \\
\hline & & $\mathrm{H}$ & 0.64785 & 2.27858 & -0.3311 & & $\mathrm{H}$ & -0.5486 & 2.1212 & -0.2342 \\
\hline & & $\mathrm{H}$ & 2.25244 & -1.5576 & -0.3275 & & $\mathrm{H}$ & 2.20652 & -1.119 & -0.3158 \\
\hline & & $\mathrm{H}$ & 2.95196 & -0.1675 & 0.68437 & & $\mathrm{H}$ & 2.60981 & 0.47592 & 0.56056 \\
\hline \multirow[t]{10}{*}{ IM4 } & 0 & 1 & & & & 0 & 1 & & & \\
\hline & & $\mathrm{C}$ & 1.46349 & -0.04 & $1.7 \mathrm{E}-05$ & & $\mathrm{C}$ & 0.80046 & 1.13244 & -0.2794 \\
\hline & & $\mathrm{C}$ & 1.53417 & 1.28253 & -0.0001 & & $\mathrm{C}$ & -0.1454 & 0.41617 & 0.59296 \\
\hline & & $\mathrm{C}$ & -2.1945 & 0.79935 & $-2 \mathrm{E}-05$ & & $\mathrm{C}$ & -2.5687 & -0.2504 & -0.2075 \\
\hline & & $\mathrm{C}$ & -1.25 & 0.05292 & $-2 \mathrm{E}-05$ & & $\mathrm{C}$ & -1.4613 & 0.051 & 0.14072 \\
\hline & & $\mathrm{S}$ & 0.01324 & -1.0646 & $8.9 \mathrm{E}-05$ & & $\mathrm{O}$ & 0.9737 & -0.2542 & -0.0101 \\
\hline & & $\mathrm{H}$ & 2.34642 & -0.6672 & 0.00013 & & $\mathrm{H}$ & 0.49548 & 1.37577 & -1.2908 \\
\hline & & $\mathrm{H}$ & 0.65224 & 1.9072 & -0.0003 & & $\mathrm{H}$ & -0.0491 & 0.55435 & 1.66521 \\
\hline & & $\mathrm{H}$ & -3.0283 & 1.45577 & $-1 \mathrm{E}-04$ & & $\mathrm{H}$ & 1.53296 & 1.79324 & 0.1725 \\
\hline & & $\mathrm{H}$ & 2.49914 & 1.76984 & -0.0002 & & $\mathrm{H}$ & -3.5459 & -0.5194 & -0.522 \\
\hline \multirow[t]{10}{*}{ BIM6 } & 0 & 3 & & & & 0 & 3 & & & \\
\hline & & $\mathrm{C}$ & -0.5525 & 0.60991 & 0.51365 & & $\mathrm{C}$ & -0.6124 & 0.51893 & 0.47661 \\
\hline & & $\mathrm{C}$ & 0.60358 & 0.64542 & -0.4165 & & $\mathrm{C}$ & 0.53553 & 0.49912 & -0.4926 \\
\hline & & $\mathrm{C}$ & 2.85714 & -0.5104 & 0.12029 & & $\mathrm{C}$ & 2.86263 & -0.4201 & 0.16088 \\
\hline & & $\mathrm{C}$ & 1.79675 & 0.03041 & -0.1401 & & $\mathrm{C}$ & 1.76921 & 0.01607 & -0.1585 \\
\hline & & $\mathrm{S}$ & -1.9356 & -0.4346 & -0.0924 & & $\mathrm{O}$ & -1.8007 & 0.12576 & -0.0671 \\
\hline & & $\mathrm{H}$ & -0.2742 & 0.27633 & 1.51166 & & $\mathrm{H}$ & -0.3755 & -0.0226 & 1.40465 \\
\hline & & $\mathrm{H}$ & 0.46626 & 1.12071 & -1.3795 & & $\mathrm{H}$ & 0.34444 & 0.86888 & -1.492 \\
\hline & & $\mathrm{H}$ & -1.0175 & 1.59762 & 0.61701 & & $\mathrm{H}$ & -0.8152 & 1.56349 & 0.81116 \\
\hline & & $\mathrm{H}$ & 3.78112 & -0.9838 & 0.33895 & & $\mathrm{H}$ & 3.81699 & -0.7979 & 0.42994 \\
\hline \multirow[t]{8}{*}{ TS5 } & 0 & 1 & & & & 0 & 1 & & & \\
\hline & & $\mathrm{C}$ & 0.8087 & 0.83698 & $1.5 \mathrm{E}-05$ & & $\mathrm{C}$ & -1.3321 & 0.20978 & 0.00015 \\
\hline & & $\mathrm{C}$ & -0.6703 & 1.12067 & $-5 \mathrm{E}-05$ & & $\mathrm{C}$ & -0.0154 & 0.97678 & $-2 \mathrm{E}-05$ \\
\hline & & $\mathrm{C}$ & -1.5211 & -0.0762 & $-4 \mathrm{E}-05$ & & $\mathrm{C}$ & 1.15565 & 0.09606 & -0.0001 \\
\hline & & $\mathrm{C}$ & -2.1812 & -1.1411 & $-9 \mathrm{E}-05$ & & $\mathrm{C}$ & 2.08742 & -0.7418 & -0.0003 \\
\hline & & $\mathrm{S}$ & 1.53687 & -0.6006 & $1.6 \mathrm{E}-05$ & & $\mathrm{O}$ & -1.4389 & -0.9817 & 0.00019 \\
\hline & & $\mathrm{H}$ & 1.39873 & 1.75326 & $3.3 \mathrm{E}-05$ & & $\mathrm{H}$ & -2.217 & 0.87596 & 0.00022 \\
\hline & & $\mathrm{H}$ & -0.8863 & 1.74716 & 0.87362 & & $\mathrm{H}$ & -0.0262 & 1.63385 & -0.8761 \\
\hline
\end{tabular}




\begin{tabular}{|c|c|c|c|c|c|c|c|c|c|c|}
\hline & & $\begin{array}{l}\mathrm{H} \\
\mathrm{H}\end{array}$ & $\begin{array}{l}-2.8325 \\
-0.8862\end{array}$ & $\begin{array}{l}-0.0811 \\
1.74714\end{array}$ & $\begin{array}{l}0.00078 \\
-0.8738\end{array}$ & & $\begin{array}{l}\mathrm{H} \\
\mathrm{H}\end{array}$ & $\begin{array}{c}2.40633 \\
-0.026\end{array}$ & $\begin{array}{l}0.46508 \\
1.63385\end{array}$ & $\begin{array}{c}0.00021 \\
0.8761\end{array}$ \\
\hline \multirow[t]{10}{*}{ Ts6 } & 0 & 1 & & & & 0 & 1 & & & \\
\hline & & $\mathrm{C}$ & 0.02892 & 1.2347 & -0.0278 & & $\mathrm{C}$ & 1.08835 & 0.27493 & -0.025 \\
\hline & & $\mathrm{C}$ & 1.33778 & 0.81707 & 0.02669 & & $\mathrm{C}$ & 0.77654 & -1.0569 & 0.0348 \\
\hline & & $\mathrm{C}$ & 1.07974 & -1.1463 & -0.1403 & & $\mathrm{C}$ & -1.1803 & -0.6891 & -0.1651 \\
\hline & & $\mathrm{C}$ & -0.182 & -1.3501 & 0.02793 & & $\mathrm{C}$ & -1.1454 & 0.58049 & 0.07283 \\
\hline & & $\mathrm{S}$ & -1.1911 & 0.02088 & 0.01248 & & $\mathrm{O}$ & 0.10132 & 1.16841 & 0.01632 \\
\hline & & $\mathrm{H}$ & -0.2898 & 2.25758 & -0.1718 & & $\mathrm{H}$ & 2.0594 & 0.71443 & -0.2014 \\
\hline & & $\mathrm{H}$ & 2.12972 & 1.48275 & -0.3003 & & $\mathrm{H}$ & 1.50817 & -1.7729 & -0.3219 \\
\hline & & $\mathrm{H}$ & 2.01242 & -1.6499 & 0.03483 & & $\mathrm{H}$ & -1.8276 & -1.5303 & -0.0143 \\
\hline & & $\mathrm{H}$ & 1.61906 & 0.24331 & 0.91853 & & $\mathrm{H}$ & 0.21437 & -1.4149 & 0.90141 \\
\hline \multirow[t]{10}{*}{ TS7 } & 0 & 1 & & & & 0 & 1 & & & \\
\hline & & $\mathrm{C}$ & 1.59966 & -2.1229 & 0 & & $\mathrm{C}$ & 2.05315 & 0.6869 & 0.00014 \\
\hline & & $\mathrm{C}$ & 0.41041 & -2.5038 & 0 & & $\mathrm{C}$ & 2.16026 & -0.5576 & 0.00013 \\
\hline & & $\mathrm{C}$ & -1.3091 & 2.7951 & 0 & & $\mathrm{C}$ & -2.7176 & -0.0007 & 0.00021 \\
\hline & & $\mathrm{C}$ & -0.7591 & 1.7128 & 0 & & $\mathrm{C}$ & -1.4997 & -0.0196 & $-5 \mathrm{E}-05$ \\
\hline & & $\mathrm{S}$ & 0 & 0.23454 & 0 & & $\mathrm{O}$ & -0.2539 & -0.0298 & -0.0005 \\
\hline & & $\mathrm{H}$ & 2.59058 & -1.7334 & 0 & & $\mathrm{H}$ & 1.86549 & 1.73574 & 0.0001 \\
\hline & & $\mathrm{H}$ & -0.3682 & -1.5792 & 0 & & $\mathrm{H}$ & 1.00946 & -0.8774 & $2.5 \mathrm{E}-05$ \\
\hline & & $\mathrm{H}$ & -1.7921 & 3.73929 & 0 & & $\mathrm{H}$ & -3.7757 & 0.01014 & 0.00073 \\
\hline & & $\mathrm{H}$ & -0.0816 & -3.4663 & 0 & & $\mathrm{H}$ & 2.9553 & -1.2847 & 0.00034 \\
\hline \multirow[t]{10}{*}{ TS8 } & 0 & 1 & & & & 0 & 1 & & & \\
\hline & & $\mathrm{C}$ & -0.3434 & 0.67911 & 0.5428 & & $\mathrm{C}$ & -0.6223 & 0.12349 & 0.43534 \\
\hline & & $\mathrm{C}$ & -1.0634 & 1.47544 & -0.3212 & & $\mathrm{C}$ & -1.6315 & -0.7023 & -0.2193 \\
\hline & & $\mathrm{C}$ & 2.16212 & 0.21637 & -0.1175 & & $\mathrm{C}$ & 1.94329 & -0.5099 & -0.0866 \\
\hline & & $\mathrm{C}$ & 0.95987 & 0.16102 & 0.08726 & & $\mathrm{C}$ & 0.79285 & -0.161 & 0.12322 \\
\hline & & $\mathrm{S}$ & -0.6415 & -1.2213 & -0.069 & & $\mathrm{O}$ & -0.1457 & 1.26469 & -0.2215 \\
\hline & & $\mathrm{H}$ & -0.4823 & 0.77038 & 1.61115 & & $\mathrm{H}$ & -0.7875 & 0.24914 & 1.51197 \\
\hline & & $\mathrm{H}$ & -0.8863 & 1.42063 & -1.3843 & & $\mathrm{H}$ & -1.8257 & -0.568 & -1.2732 \\
\hline & & $\mathrm{H}$ & 3.19006 & 0.0326 & -0.3112 & & $\mathrm{H}$ & 2.95402 & -0.7621 & -0.2895 \\
\hline & & $\mathrm{H}$ & -1.8487 & 2.12514 & 0.04085 & & $\mathrm{H}$ & -2.0692 & -1.538 & 0.30675 \\
\hline \multirow[t]{10}{*}{ TS9 } & 0 & 1 & & & & 0 & 1 & & & \\
\hline & & $\mathrm{C}$ & 0.87678 & 0.37547 & 0.01149 & & $\mathrm{C}$ & 0.93632 & 0.3002 & 0.02132 \\
\hline & & $\mathrm{C}$ & -0.7338 & 1.17883 & -0.0097 & & $\mathrm{C}$ & -0.8308 & 1.2799 & -0.0143 \\
\hline & & $\mathrm{C}$ & -1.5849 & 0.0703 & -0.0101 & & $\mathrm{C}$ & -1.5083 & 0.08398 & -0.0162 \\
\hline & & $\mathrm{C}$ & -1.2936 & -1.1675 & 0.0019 & & $\mathrm{C}$ & -1.2534 & -1.1433 & -0.0021 \\
\hline & & $\mathrm{S}$ & 2.23859 & 1.15223 & 0.01631 & & $\mathrm{O}$ & 1.99604 & 0.74312 & 0.02959 \\
\hline & & $\mathrm{H}$ & -0.0679 & -1.0557 & 0.01325 & & $\mathrm{H}$ & 0.15987 & -0.8621 & 0.01957 \\
\hline & & $\mathrm{H}$ & -0.7295 & 1.80254 & 0.88301 & & $\mathrm{H}$ & -0.8272 & 1.88275 & 0.88935 \\
\hline & & $\mathrm{H}$ & -1.7736 & -2.1281 & 0.00437 & & $\mathrm{H}$ & -1.6553 & -2.1352 & -0.0008 \\
\hline & & $\mathrm{H}$ & -0.7122 & 1.79014 & -0.9106 & & $\mathrm{H}$ & -0.7973 & 1.86882 & -0.9265 \\
\hline \multirow[t]{10}{*}{ TS10 } & 0 & 1 & & & & 0 & 1 & & & \\
\hline & & $\mathrm{C}$ & -0.9639 & -0.7558 & 0.00009 & & $\mathrm{C}$ & -1.3983 & -0.5914 & 0.00013 \\
\hline & & $\mathrm{C}$ & 0.8612 & 0.75719 & $1.5 \mathrm{E}-05$ & & $\mathrm{C}$ & 0.27771 & 0.94774 & $4.5 \mathrm{E}-05$ \\
\hline & & $\mathrm{C}$ & 2.14375 & 0.19159 & -0.0002 & & $\mathrm{C}$ & 1.48965 & 0.22613 & $-3 E-05$ \\
\hline & & $\mathrm{C}$ & 3.20713 & -0.3852 & -0.0003 & & $\mathrm{C}$ & 2.41221 & -0.5581 & $-8 \mathrm{E}-05$ \\
\hline & & $\mathrm{S}$ & -2.3008 & 0.02618 & 0.00017 & & $\mathrm{O}$ & -2.4638 & -0.1525 & -0.0001 \\
\hline & & $\mathrm{H}$ & 0.12487 & -0.8754 & $2.9 \mathrm{E}-05$ & & $\mathrm{H}$ & -0.3159 & -0.7386 & 0.00068 \\
\hline & & $\mathrm{H}$ & 0.51948 & 1.23042 & -0.9122 & & $\mathrm{H}$ & 0.0248 & 1.48313 & -0.9101 \\
\hline & & $\mathrm{H}$ & 4.1598 & -0.8513 & -0.0005 & & $\mathrm{H}$ & 3.28934 & -1.1545 & $-6 \mathrm{E}-05$ \\
\hline & & $\mathrm{H}$ & 0.5197 & 1.2303 & 0.91233 & & $\mathrm{H}$ & 0.02502 & 1.48326 & 0.91016 \\
\hline \multirow[t]{10}{*}{ TS11 } & 0 & 1 & & & & 0 & 1 & & & \\
\hline & & $\mathrm{C}$ & 0.56985 & 0.2155 & 0.38851 & & $\mathrm{C}$ & 1.13145 & -0.1265 & 0.29208 \\
\hline & & $\mathrm{C}$ & -0.6008 & 0.98865 & -0.1179 & & $\mathrm{C}$ & -0.0148 & 0.86274 & -0.0033 \\
\hline & & $\mathrm{C}$ & -1.5795 & -0.0968 & 0.21626 & & $\mathrm{C}$ & -1.0821 & -0.1735 & 0.18433 \\
\hline & & $\mathrm{C}$ & -2.6736 & -0.5312 & -0.2458 & & $\mathrm{C}$ & -2.2735 & -0.256 & -0.2157 \\
\hline & & $\mathrm{S}$ & 1.96338 & -0.2978 & -0.1096 & & $\mathrm{O}$ & 2.13328 & -0.3835 & -0.2415 \\
\hline & & $\mathrm{H}$ & -0.9243 & -0.6067 & 1.09266 & & $\mathrm{H}$ & -0.4235 & -0.9828 & 0.81011 \\
\hline & & $\mathrm{H}$ & -0.7943 & 1.83748 & 0.54649 & & $\mathrm{H}$ & -0.0534 & 1.59781 & 0.80603 \\
\hline & & $\mathrm{H}$ & -3.4487 & -1.267 & -0.1782 & & $\mathrm{H}$ & -3.2137 & -0.7628 & -0.2602 \\
\hline & & $\mathrm{H}$ & -0.5425 & 1.34312 & -1.1538 & & $\mathrm{H}$ & 0.05806 & 1.37507 & -0.9686 \\
\hline \multirow[t]{4}{*}{ TS12 } & 0 & 1 & & & & 0 & 1 & & & \\
\hline & & $\mathrm{C}$ & -0.7924 & 1.30049 & 0.07223 & & $\mathrm{C}$ & 1.06336 & -0.8222 & 0.03381 \\
\hline & & $\mathrm{C}$ & -1.4913 & 0.08828 & -0.0446 & & $\mathrm{C}$ & 0.98795 & 0.59937 & 0.0041 \\
\hline & & $\mathrm{C}$ & -0.8671 & -1.158 & -0.0011 & & $\mathrm{C}$ & -0.2415 & 1.19148 & -0.0055 \\
\hline
\end{tabular}




\begin{tabular}{|c|c|c|c|c|c|c|c|c|c|c|}
\hline & & $\mathrm{C}$ & 0.51379 & -1.1172 & 0.16361 & & $\mathrm{C}$ & -1.2724 & 0.18599 & 0.07203 \\
\hline & & $\mathrm{S}$ & 1.32809 & 0.2228 & -0.0813 & & $\mathrm{O}$ & -0.9165 & -0.9646 & -0.1012 \\
\hline & & $\mathrm{H}$ & -1.1965 & 2.18098 & -0.4145 & & $\mathrm{H}$ & 1.74871 & -1.3277 & -0.6389 \\
\hline & & $\mathrm{H}$ & -2.5479 & 0.11587 & -0.2906 & & $\mathrm{H}$ & 1.87874 & 1.20444 & -0.1161 \\
\hline & & $\mathrm{H}$ & -1.4253 & -2.0745 & -0.1255 & & $\mathrm{H}$ & -0.4259 & 2.25221 & -0.0336 \\
\hline & & $\mathrm{H}$ & -0.2574 & 1.53105 & 0.99011 & & $\mathrm{H}$ & 0.90648 & -1.3405 & 0.97169 \\
\hline TS13 & 0 & 1 & & & & 0 & 1 & & & \\
\hline & & $\mathrm{C}$ & 2.53949 & -0.7184 & 0.13552 & & $\mathrm{C}$ & -1.998 & -0.495 & 0.021 \\
\hline & & $\mathrm{C}$ & 1.91818 & 0.45123 & -0.201 & & $\mathrm{C}$ & -1.139 & 0.535 & -0.064 \\
\hline & & $\mathrm{C}$ & 0.57561 & 0.53174 & 0.01906 & & $\mathrm{C}$ & 0.205 & 0.568 & 0.283 \\
\hline & & $\mathrm{C}$ & -0.7063 & 0.6092 & 0.25146 & & $\mathrm{C}$ & 1.495 & 0.412 & -0.027 \\
\hline & & $\mathrm{S}$ & -2.0651 & -0.3437 & -0.1075 & & $\mathrm{O}$ & 1.558 & -0.857 & -0.092 \\
\hline & & $\mathrm{H}$ & 3.48176 & -0.9941 & -0.3191 & & $\mathrm{H}$ & -3.006 & -0.391 & -0.349 \\
\hline & & $\mathrm{H}$ & 2.42274 & 1.18675 & -0.8217 & & $\mathrm{H}$ & -1.483 & 1.484 & -0.479 \\
\hline & & $\mathrm{H}$ & 2.04388 & -1.4472 & 0.76034 & & $\mathrm{H}$ & -1.682 & -1.46 & 0.388 \\
\hline & & $\mathrm{H}$ & -0.8689 & 1.51096 & 0.87068 & & $\mathrm{H}$ & 2.328 & 1.111 & -0.105 \\
\hline TS14 & 0 & 1 & & & & 0 & 1 & & & \\
\hline & & $\mathrm{C}$ & -1.7037 & -1.0675 & -0.028 & & $\mathrm{C}$ & -1.5162 & -0.8163 & -0.028 \\
\hline & & $\mathrm{C}$ & -1.7855 & 0.3159 & -0.1225 & & $\mathrm{C}$ & -1.2394 & 0.54381 & -0.1199 \\
\hline & & $\mathrm{C}$ & -0.6485 & 1.22419 & -0.0002 & & $\mathrm{C}$ & 0.09283 & 1.09566 & 0.00021 \\
\hline & & $\mathrm{C}$ & 0.52697 & 0.63093 & 0.01671 & & $\mathrm{C}$ & 1.07982 & 0.20852 & 0.01383 \\
\hline & & $\mathrm{S}$ & 1.75462 & -0.3299 & -0.001 & & $\mathrm{O}$ & 1.79647 & -0.7009 & 0.00018 \\
\hline & & $\mathrm{H}$ & -2.6125 & -1.6392 & -0.1888 & & $\mathrm{H}$ & -2.5395 & -1.141 & -0.1868 \\
\hline & & $\mathrm{H}$ & -2.2161 & -0.33 & 0.92562 & & $\mathrm{H}$ & -1.8377 & 0.0347 & 0.91859 \\
\hline & & $\mathrm{H}$ & -0.7942 & 2.29365 & 0.03021 & & $\mathrm{H}$ & 0.28637 & 2.15613 & 0.02775 \\
\hline & & $\mathrm{H}$ & -0.7867 & -1.6668 & 0.05268 & & $\mathrm{H}$ & -0.7835 & -1.6324 & 0.04208 \\
\hline TS15 & 0 & 1 & & & & & & & & \\
\hline & & $\mathrm{C}$ & 1.67174 & -1.1326 & $8.4 \mathrm{E}-05$ & & & & & \\
\hline & & $\mathrm{C}$ & 1.55274 & 0.29764 & $2.1 \mathrm{E}-05$ & & & & & \\
\hline & & $\mathrm{C}$ & 0.96896 & 1.38395 & $-1 \mathrm{E}-05$ & & & & & \\
\hline & & $\mathrm{H}$ & 2.18839 & -1.5036 & 0.88617 & & & & & \\
\hline & & $\mathrm{H}$ & 2.18838 & -1.5037 & -0.886 & & & & & \\
\hline & & $\mathrm{H}$ & 1.00489 & 2.44835 & $-9 E-06$ & & & & & \\
\hline & & $\mathrm{H}$ & 0.64377 & -1.5578 & 0.00011 & & & & & \\
\hline & & $\mathrm{C}$ & -0.9519 & 0.98697 & $-6 E-05$ & & & & & \\
\hline & & $\mathrm{S}$ & -1.5921 & -0.4437 & $-3 \mathrm{E}-05$ & & & & & \\
\hline TS16 & 0 & 1 & & & & 0 & 1 & & & \\
\hline & & $\mathrm{C}$ & 1.93053 & -0.9942 & -0.028 & & $\mathrm{C}$ & 1.56568 & -0.8603 & -0.023 \\
\hline & & $\mathrm{C}$ & 1.81588 & 0.31885 & 0.06959 & & $\mathrm{C}$ & 1.3237 & 0.42988 & 0.15137 \\
\hline & & $\mathrm{C}$ & 0.61855 & 1.23049 & 0.12922 & & $\mathrm{C}$ & -0.0721 & 1.07863 & 0.14976 \\
\hline & & $\mathrm{C}$ & -0.5368 & 0.5008 & 0.05524 & & $\mathrm{C}$ & -1.0126 & 0.09067 & 0.04626 \\
\hline & & $\mathrm{S}$ & -1.391 & -0.7924 & 0.14164 & & $\mathrm{O}$ & -1.4685 & -0.9585 & 0.09207 \\
\hline & & $\mathrm{H}$ & 2.87298 & -1.484 & 0.18373 & & $\mathrm{H}$ & 2.56476 & -1.2434 & 0.14243 \\
\hline & & $\mathrm{H}$ & 1.32289 & 1.0823 & 1.13069 & & $\mathrm{H}$ & 0.60598 & 1.08737 & 1.15713 \\
\hline & & $\mathrm{H}$ & 0.7205 & 2.27697 & -0.1124 & & $\mathrm{H}$ & -0.2236 & 2.09614 & -0.1636 \\
\hline & & $\mathrm{H}$ & 1.11184 & -1.6508 & -0.3506 & & $\mathrm{H}$ & 0.82779 & -1.5926 & -0.3662 \\
\hline TS17 & 0 & 1 & & & & & & & & \\
\hline & & $\mathrm{C}$ & 1.50846 & -1.2708 & $-1 \mathrm{E}-06$ & & & & & \\
\hline & & $\mathrm{C}$ & 1.59292 & 0.0141 & $-3 E-06$ & & & & & \\
\hline & & $\mathrm{C}$ & 1.04976 & 1.26008 & 7E-06 & & & & & \\
\hline & & $\mathrm{C}$ & -0.8113 & 0.99372 & $-2 \mathrm{E}-05$ & & & & & \\
\hline & & $\mathrm{S}$ & -1.5701 & -0.377 & $4 \mathrm{E}-06$ & & & & & \\
\hline & & $\mathrm{H}$ & 2.33518 & -1.9672 & $-1 \mathrm{E}-06$ & & & & & \\
\hline & & $\mathrm{H}$ & 1.13311 & 1.85555 & -0.9028 & & & & & \\
\hline & & $\mathrm{H}$ & 1.13307 & 1.8555 & 0.90284 & & & & & \\
\hline & & $\mathrm{H}$ & 0.48163 & -1.6939 & $-2 \mathrm{E}-05$ & & & & & \\
\hline IM6 & 0 & 1 & & & & 0 & 1 & & & \\
\hline & & $\mathrm{C}$ & 2.11942 & -0.979 & $-4 \mathrm{E}-06$ & & $\mathrm{C}$ & 1.74455 & -0.8207 & $1.9 \mathrm{E}-05$ \\
\hline & & $\mathrm{C}$ & 1.88473 & 0.33453 & 0.00015 & & $\mathrm{C}$ & 1.30528 & 0.43843 & 0.00017 \\
\hline & & $\mathrm{C}$ & 0.58313 & 0.99932 & $-8 \mathrm{E}-05$ & & $\mathrm{C}$ & -0.0782 & 0.9085 & 3E-06 \\
\hline & & $\mathrm{C}$ & -0.5929 & 0.41313 & $-5 \mathrm{E}-06$ & & $\mathrm{C}$ & -1.1407 & 0.12968 & $-6 E-05$ \\
\hline & & $\mathrm{S}$ & -1.9821 & -0.29 & $-4 \mathrm{E}-05$ & & $\mathrm{O}$ & -2.0758 & -0.555 & -0.0001 \\
\hline & & $\mathrm{H}$ & 3.13127 & -1.3571 & 0.00024 & & $\mathrm{H}$ & 2.80333 & -1.0329 & 0.0002 \\
\hline & & $\mathrm{H}$ & 2.72718 & 1.01582 & 0.00049 & & $\mathrm{H}$ & 2.032 & 1.24199 & 0.00045 \\
\hline & & $\mathrm{H}$ & 0.56725 & 2.08479 & -0.0002 & & $\mathrm{H}$ & -0.2908 & 1.96905 & -0.0001 \\
\hline & & $\mathrm{H}$ & 1.32216 & -1.7109 & -0.0004 & & $\mathrm{H}$ & 1.07643 & -1.6732 & -0.0003 \\
\hline
\end{tabular}




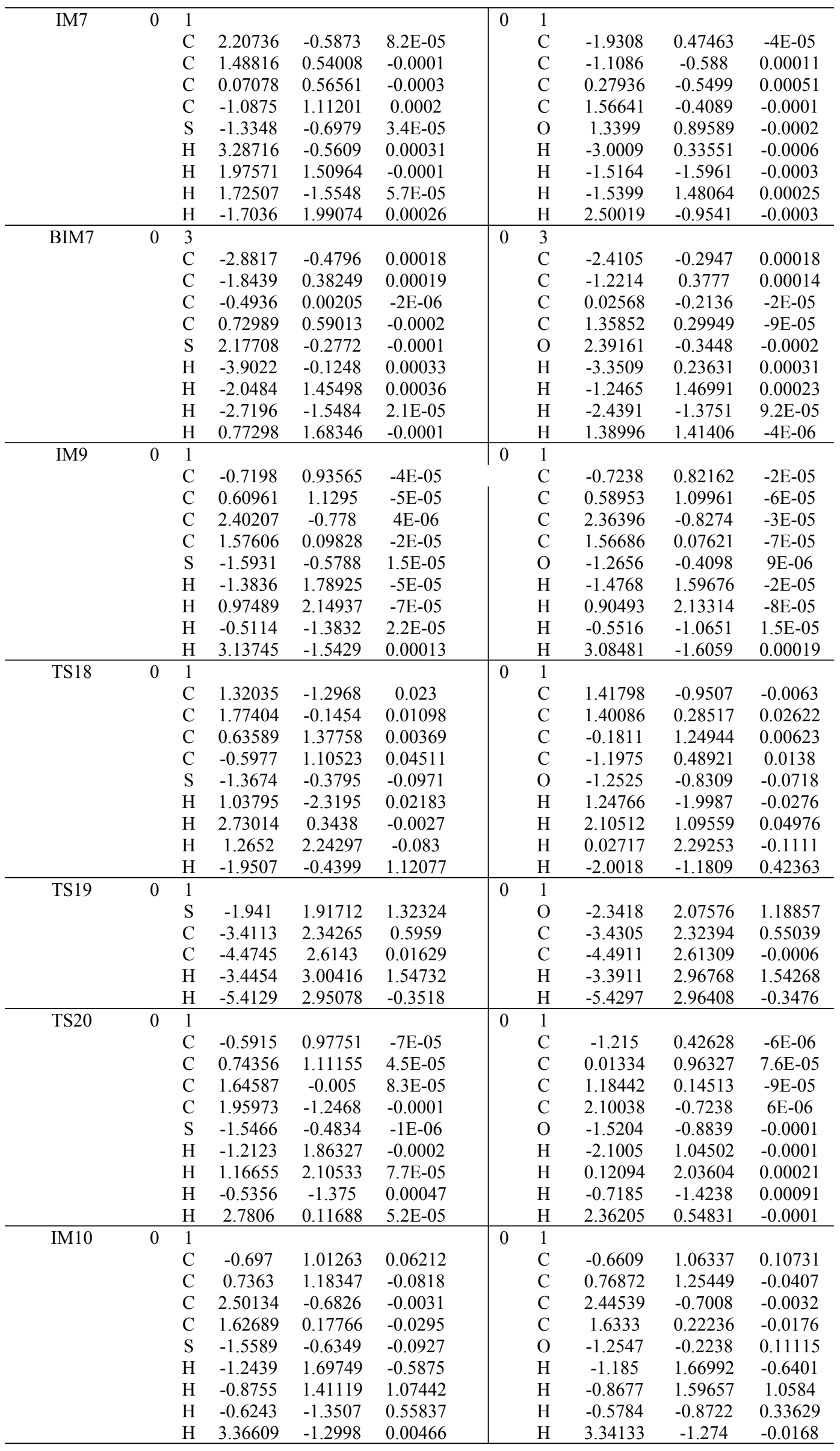




\begin{tabular}{|c|c|c|c|c|c|c|c|c|c|c|}
\hline TS21 & 0 & $\begin{array}{l}1 \\
\mathrm{C} \\
\mathrm{C} \\
\mathrm{C} \\
\mathrm{C} \\
\mathrm{S} \\
\mathrm{H} \\
\mathrm{H} \\
\mathrm{H} \\
\mathrm{H}\end{array}$ & $\begin{array}{l}0.75371 \\
-0.4445 \\
-1.6442 \\
-2.7844 \\
1.82053 \\
1.14295 \\
-0.6833 \\
-3.7799 \\
-1.0918 \\
\end{array}$ & $\begin{array}{c}0.75954 \\
1.25767 \\
-0.7519 \\
-0.3716 \\
-0.4967 \\
1.8285 \\
-1.2356 \\
-0.0017 \\
1.99445 \\
\end{array}$ & $\begin{array}{c}0.01444 \\
-0.096 \\
-0.0298 \\
0.02476 \\
0.01346 \\
-0.0327 \\
-0.0606 \\
0.06102 \\
0.33642 \\
\end{array}$ & & & & & \\
\hline TS22 & 0 & $\begin{array}{l}1 \\
\mathrm{C} \\
\mathrm{C} \\
\mathrm{C} \\
\mathrm{C} \\
\mathrm{S} \\
\mathrm{H} \\
\mathrm{H} \\
\mathrm{H} \\
\mathrm{H}\end{array}$ & $\begin{array}{l}-0.6745 \\
0.70127 \\
2.44534 \\
1.59247 \\
-1.6013 \\
-1.3491 \\
-0.1273 \\
-0.5156 \\
3.22463 \\
\end{array}$ & $\begin{array}{c}0.96229 \\
1.17969 \\
-0.7375 \\
0.12431 \\
-0.5908 \\
1.79532 \\
1.332 \\
-1.3896 \\
-1.4574 \\
\end{array}$ & $\begin{array}{c}-0.0077 \\
-0.1286 \\
0.0183 \\
-0.0152 \\
-0.0045 \\
-0.1801 \\
1.02566 \\
-0.0207 \\
0.0461 \\
\end{array}$ & 0 & $\begin{array}{l}1 \\
\mathrm{C} \\
\mathrm{C} \\
\mathrm{C} \\
\mathrm{C} \\
\mathrm{O} \\
\mathrm{H} \\
\mathrm{H} \\
\mathrm{H} \\
\mathrm{H}\end{array}$ & $\begin{array}{l}1.23674 \\
-0.0216 \\
-2.1844 \\
-1.1494 \\
1.51141 \\
2.13216 \\
0.93998 \\
0.67339 \\
-3.1248 \\
\end{array}$ & $\begin{array}{c}-0.4228 \\
-1.0388 \\
0.39345 \\
-0.2418 \\
0.92975 \\
-0.9832 \\
-0.8929 \\
1.41211 \\
0.88498 \\
\end{array}$ & $\begin{array}{c}0.00637 \\
-0.1268 \\
0.01942 \\
-0.0154 \\
-0.0204 \\
-0.2441 \\
1.07054 \\
-0.0115 \\
0.0469 \\
\end{array}$ \\
\hline TS23 & 0 & $\begin{array}{l}1 \\
\mathrm{C} \\
\mathrm{C} \\
\mathrm{C} \\
\mathrm{C} \\
\mathrm{S} \\
\mathrm{H} \\
\mathrm{H} \\
\mathrm{H} \\
\mathrm{H}\end{array}$ & $\begin{array}{c}-0.3618 \\
0.93338 \\
2.82736 \\
1.89077 \\
-2.0342 \\
-0.9074 \\
-0.8046 \\
-1.1032 \\
3.6239 \\
\end{array}$ & $\begin{array}{c}1.17592 \\
0.9412 \\
-0.7695 \\
0.01412 \\
-0.5024 \\
1.97604 \\
0.82953 \\
-1.4651 \\
-1.4715 \\
\end{array}$ & $\begin{array}{c}0.0298 \\
-0.0486 \\
-0.0035 \\
-0.0065 \\
-0.0305 \\
-0.4423 \\
0.99971 \\
0.10496 \\
-0.0011 \\
\end{array}$ & 0 & $\begin{array}{l}1 \\
\mathrm{C} \\
\mathrm{C} \\
\mathrm{C} \\
\mathrm{C} \\
\mathrm{O} \\
\mathrm{H} \\
\mathrm{H} \\
\mathrm{H} \\
\mathrm{H}\end{array}$ & $\begin{array}{c}1.06289 \\
-0.2435 \\
-2.4832 \\
-1.3911 \\
2.1284 \\
1.78103 \\
1.46313 \\
1.47078 \\
-3.4133 \\
\end{array}$ & $\begin{array}{c}-0.7504 \\
-0.7849 \\
0.44585 \\
-0.0954 \\
0.78772 \\
-1.4259 \\
-0.221 \\
1.49707 \\
0.95695 \\
\end{array}$ & $\begin{array}{c}0.00249 \\
-0.0049 \\
-0.0084 \\
-0.0006 \\
-0.043 \\
-0.43 \\
0.93454 \\
-0.0686 \\
-0.0232 \\
\end{array}$ \\
\hline TS24 & 0 & $\begin{array}{l}1 \\
\mathrm{C} \\
\mathrm{C} \\
\mathrm{C} \\
\mathrm{C} \\
\mathrm{S} \\
\mathrm{H} \\
\mathrm{H} \\
\mathrm{H} \\
\mathrm{H}\end{array}$ & $\begin{array}{c}0.33911 \\
-0.575 \\
-1.9428 \\
-3.1523 \\
2.01023 \\
0.31672 \\
2.42709 \\
-4.2083 \\
1.28601 \\
\end{array}$ & $\begin{array}{c}0.62205 \\
-0.3517 \\
-0.108 \\
-0.0218 \\
-0.0754 \\
1.70635 \\
-0.1332 \\
0.07021 \\
-1.2807 \\
\end{array}$ & $\begin{array}{c}0.0119 \\
0.00856 \\
0.00484 \\
0.00562 \\
-0.0837 \\
-0.0145 \\
1.21242 \\
0.00395 \\
-0.0489 \\
\end{array}$ & & & & & \\
\hline TS25 & 0 & $\begin{array}{l}1 \\
\mathrm{C} \\
\mathrm{C} \\
\mathrm{C} \\
\mathrm{C} \\
\mathrm{S} \\
\mathrm{H} \\
\mathrm{H} \\
\mathrm{H} \\
\mathrm{H}\end{array}$ & $\begin{array}{c}-0.7772 \\
0.60788 \\
2.46762 \\
1.56755 \\
-1.5765 \\
-0.744 \\
0.92515 \\
-1.382 \\
3.23007\end{array}$ & $\begin{array}{l}0.91423 \\
1.03636 \\
-0.7426 \\
0.05864 \\
-0.6754 \\
0.96694 \\
1.94732 \\
1.76339 \\
-1.4716\end{array}$ & $\begin{array}{l}0.23272 \\
-0.1918 \\
0.08293 \\
-0.0513 \\
-0.0751 \\
1.33859 \\
-0.6926 \\
-0.0851 \\
0.20591\end{array}$ & 0 & $\begin{array}{l}1 \\
\mathrm{C} \\
\mathrm{C} \\
\mathrm{C} \\
\mathrm{C} \\
\mathrm{O} \\
\mathrm{H} \\
\mathrm{H} \\
\mathrm{H} \\
\mathrm{H}\end{array}$ & $\begin{array}{c}-1.3741 \\
-0.0576 \\
2.22168 \\
1.13177 \\
-1.3656 \\
-1.637 \\
-0.0202 \\
-2.1091 \\
3.16034\end{array}$ & $\begin{array}{c}0.25259 \\
0.86802 \\
-0.3428 \\
0.17524 \\
-1.0108 \\
0.49764 \\
1.8351 \\
0.86197 \\
-0.8264\end{array}$ & $\begin{array}{l}0.21305 \\
-0.1026 \\
0.06841 \\
-0.0404 \\
-0.1647 \\
1.27554 \\
-0.6003 \\
-0.3708 \\
0.18271\end{array}$ \\
\hline $\begin{array}{c}\mathrm{P} 1 \\
\mathrm{SCCH} 2\end{array}$ & 0 & $\begin{array}{l}1 \\
\mathrm{~S} \\
\mathrm{C} \\
\mathrm{C} \\
\mathrm{H} \\
\mathrm{H}\end{array}$ & $\begin{array}{l}1.11286 \\
-0.4479 \\
-1.7516 \\
-2.3044 \\
-2.3047\end{array}$ & $\begin{array}{c}9 \mathrm{E}-06 \\
-7 \mathrm{E}-05 \\
1.5 \mathrm{E}-05 \\
0.93 \\
-0.9298\end{array}$ & $\begin{array}{c}-9 \mathrm{E}-05 \\
5.5 \mathrm{E}-05 \\
0.00013 \\
0.00019 \\
0.00019\end{array}$ & 0 & $\begin{array}{l}1 \\
\mathrm{O} \\
\mathrm{C} \\
\mathrm{C} \\
\mathrm{H} \\
\mathrm{H}\end{array}$ & $\begin{array}{l}1.26248 \\
0.10208 \\
-1.2058 \\
-1.7389 \\
-1.7388 \\
\end{array}$ & $\begin{array}{c}-2 \mathrm{E}-06 \\
9 \mathrm{E}-06 \\
-1 \mathrm{E}-06 \\
0.93696 \\
-0.937\end{array}$ & $\begin{array}{c}-0.0001 \\
-8 \mathrm{E}-06 \\
9.6 \mathrm{E}-05 \\
0.00014 \\
0.00014\end{array}$ \\
\hline $\mathrm{C} 2 \mathrm{H} 2$ & 0 & $\begin{array}{l}1 \\
\mathrm{C} \\
\mathrm{C} \\
\mathrm{H} \\
\mathrm{H}\end{array}$ & $\begin{array}{l}0 \\
0 \\
0 \\
0 \\
\end{array}$ & $\begin{array}{l}0 \\
0 \\
0 \\
0\end{array}$ & $\begin{array}{c}0.59794 \\
-0.5979 \\
1.65956 \\
-1.6596 \\
\end{array}$ & & & & & \\
\hline $\begin{array}{c}\mathrm{P} 2 \\
\mathrm{CH} 3 \mathrm{C} 2 \mathrm{H}\end{array}$ & 0 & $\begin{array}{l}1 \\
\mathrm{C} \\
\mathrm{C}\end{array}$ & $\begin{array}{l}-5.9402 \\
-4.4911\end{array}$ & $\begin{array}{l}0.59638 \\
0.72635\end{array}$ & $\begin{array}{l}0.00016 \\
8.3 \mathrm{E}-05\end{array}$ & & & & & \\
\hline
\end{tabular}




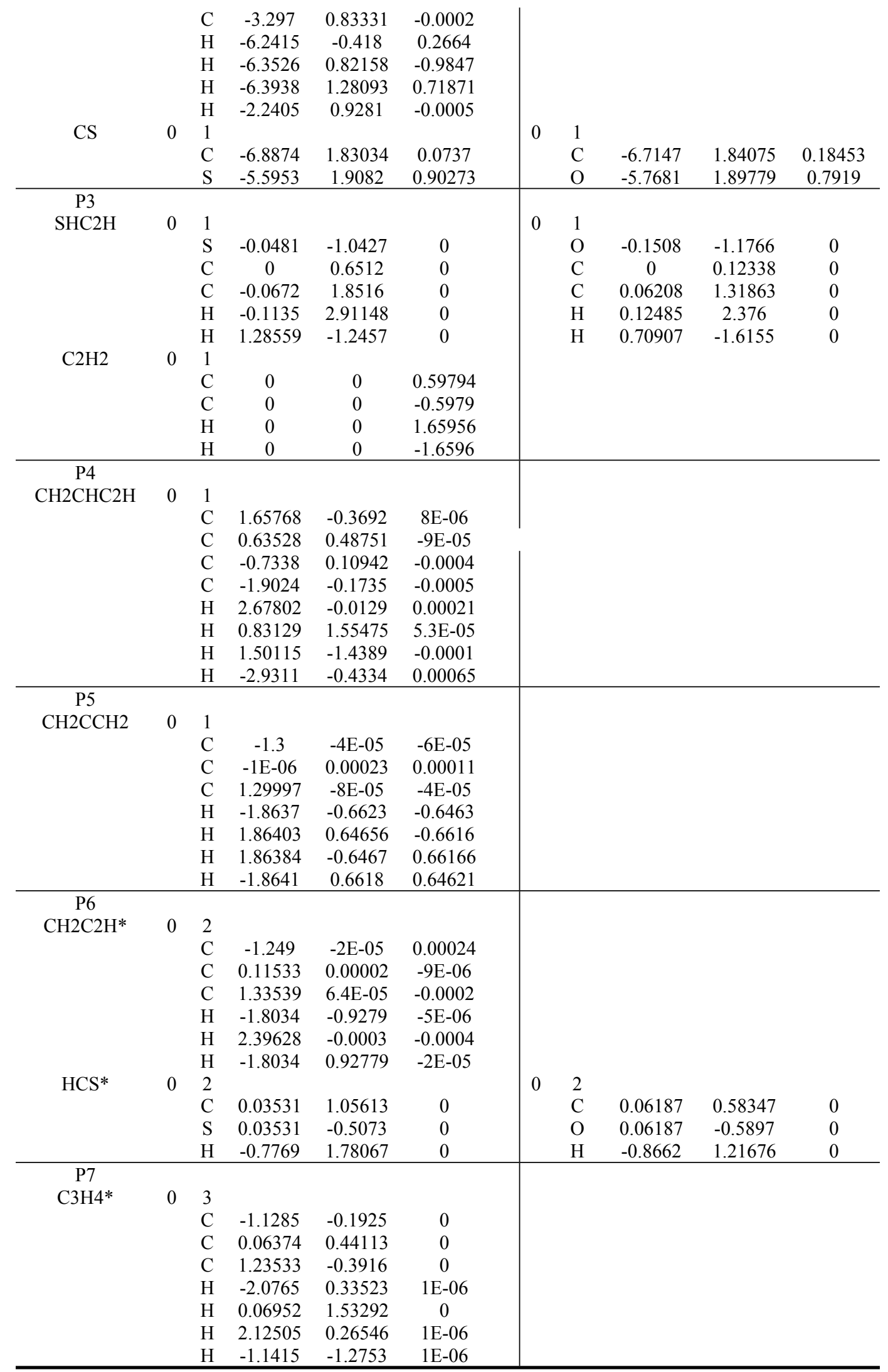

\section{VTST on CCSD(T)/CBS level}

Rate constant was determined using VTST method on CCSD $(T) / C B S$ level. The 
procedures can be described as:

(1) IRC calculation using small stepsize at B3LYP/cc-pVTZ level.

(2)Extract six geometries on each side of transition state (total 12 geometries).

(3) Do CCSD(T)/CBS extrapolation on every geometry obtained in (2).

(4) Frequency calculation of every geometry obtained in (2) to gain Gibbs correction.

(5)Locate the maximum Gibbs free energy and calculate rate constant using TST equation.

Taking the 2,3-H shift of thiophene (thiophene $\rightarrow C_{2,3}$-carbene) as an example. The $F$ in the first column indicates this point on the forward side of reaction, whereas $R$ indicates on reversed side of reaction. The Gibbs free energies of every point Rl R6 and F1 F6 calcuated at CCSD(T)/CBS//B3LYP/cc-pVTZ level is shown in Table S7.

Table S7 The CCSD(T) Gibbs free energy of the geometries.

\begin{tabular}{ccccccc}
\hline Temp. & 700 & 800 & 900 & 1000 & 1100 & 1200 \\
\hline F6 & -552.2731081838 & -552.2875941838 & -552.3027591838 & -552.3185581838 & -552.3349501838 & -552.3518981838 \\
F5 & -552.2730913912 & -552.2875773912 & -552.3027423912 & -552.3185403912 & -552.3349323912 & -552.3518803912 \\
F4 & -552.2730770470 & -552.2875630470 & -552.3027270470 & -552.3185260470 & -552.3349170470 & -552.3518650470 \\
F3 & -552.2730672336 & -552.2875522336 & -552.3027162336 & -552.3185142336 & -552.3349052336 & -552.3518542336 \\
F2 & -552.2730592498 & -552.2875442498 & -552.3027082498 & -552.3185062498 & -552.3348972498 & -552.3518452498 \\
F1 & -552.2730559953 & -552.2875409953 & -552.3027049953 & -552.3185029953 & -552.3348939953 & -552.3518419953 \\
TS & -552.2730541274 & -552.2875381274 & -552.3027011274 & -552.3184991274 & -552.3348901274 & -552.3518381274 \\
R1 & -552.2730540161 & -552.2875380161 & -552.3027010161 & -552.3184980161 & -552.3348890161 & -552.3518370161 \\
R2 & -552.2730561340 & -552.2875391340 & -552.3027021340 & -552.3184991340 & -552.3348891340 & -552.3518361340 \\
R3 & -552.2730619163 & -552.2875449163 & -552.3027069163 & -552.3185029163 & -552.3348929163 & -552.3518399163 \\
R4 & -552.2730717400 & -552.2875537400 & -552.3027157400 & -552.3185107400 & -552.3349007400 & -552.3518467400 \\
R5 & -552.2730846324 & -552.2875666324 & -552.3027276324 & -552.3185226324 & -552.3349116324 & -552.3518576324 \\
R6 & -552.2731016006 & -552.2875826006 & -552.3027436006 & -552.3185386006 & -552.3349266006 & -552.3518726006 \\
\hline & & & & & &
\end{tabular}

\begin{tabular}{ccccccc}
\hline Temp. & 1300 & 1400 & 1500 & 1600 & 1700 & 1800 \\
\hline F6 & -552.3693701838 & -552.3873371838 & -552.4057721838 & -552.4246501838 & -552.4439501838 & -552.4636511838 \\
F5 & -552.3693523912 & -552.3873193912 & -552.4057543912 & -552.4246323912 & -552.4439323912 & -552.4636333912 \\
F4 & -552.3693380470 & -552.3873050470 & -552.4057390470 & -552.4246180470 & -552.4439180470 & -552.4636190470 \\
F3 & -552.3693262336 & -552.3872932336 & -552.4057282336 & -552.4246062336 & -552.4439062336 & -552.4636072336 \\
F2 & -552.3693182498 & -552.3872842498 & -552.4057192498 & -552.4245982498 & -552.4438982498 & -552.4635992498 \\
F1 & -552.3693139953 & -552.3872809953 & -552.4057159953 & -552.4245949953 & -552.4438949953 & -552.4635969953 \\
TS & -552.3693101274 & -552.3872771274 & -552.4057121274 & -552.4245901274 & -552.4438901274 & -552.4635921274 \\
R1 & -552.3693090161 & -552.3872750161 & -552.4057100161 & -552.4245890161 & -552.4438890161 & -552.4635900161 \\
R2 & -552.3693081340 & -552.3872741340 & -552.4057081340 & -552.4245861340 & -552.4438861340 & -552.4635871340 \\
R3 & -552.3693109163 & -552.3872769163 & -552.4057109163 & -552.4245889163 & -552.4438879163 & -552.4635899163 \\
R4 & -552.3693177400 & -552.3872837400 & -552.4057177400 & -552.4245947400 & -552.4438947400 & -552.4635947400 \\
R5 & -552.3693286324 & -552.3872936324 & -552.4057276324 & -552.4246046324 & -552.4439036324 & -552.4636046324 \\
R6 & -552.3693426006 & -552.3873076006 & -552.4057406006 & -552.4246186006 & -552.4439166006 & -552.4636176006 \\
\hline
\end{tabular}

\begin{tabular}{ccccccc}
\hline Temp. & 1900 & 2000 & 2100 & 2200 & 2300 & 2400 \\
\hline F6 & -552.4837351838 & -552.5041851838 & -552.5249861838 & -552.5461221838 & -552.5675811838 & -552.4837351838 \\
F5 & -552.4837183912 & -552.5041683912 & -552.5249683912 & -552.5461053912 & -552.5675643912 & -552.4837183912 \\
F4 & -552.4837030470 & -552.5041540470 & -552.5249540470 & -552.5460910470 & -552.5675500470 & -552.4837030470 \\
F3 & -552.4836922336 & -552.5041422336 & -552.5249432336 & -552.5460802336 & -552.5675392336 & -552.4836922336 \\
F2 & -552.4836842498 & -552.5041342498 & -552.5249352498 & -552.5460722498 & -552.5675322498 & -552.4836842498 \\
F1 & -552.4836819953 & -552.5041319953 & -552.5249329953 & -552.5460709953 & -552.5675299953 & -552.4836819953 \\
TS & -552.4836771274 & -552.5041271274 & -552.5249281274 & -552.5460661274 & -552.5675251274 & -552.4836771274 \\
R1 & -552.4836750161 & -552.5041250161 & -552.5249270161 & -552.5460640161 & -552.5675230161 & -552.4836750161
\end{tabular}




\begin{tabular}{ccccccc} 
R2 & -552.4836721340 & -552.5041221340 & -552.5249231340 & -552.5460601340 & -552.5675201340 & -552.4836721340 \\
R3 & -552.4836739163 & -552.5041239163 & -552.5249249163 & -552.5460609163 & -552.5675209163 & -552.4836739163 \\
R4 & -552.4836787400 & -552.5041287400 & -552.5249297400 & -552.5460667400 & -552.5675257400 & -552.4836787400 \\
R5 & -552.4836886324 & -552.5041386324 & -552.5249386324 & -552.5460746324 & -552.5675346324 & -552.4836886324 \\
R6 & -552.4837006006 & -552.5041506006 & -552.5249506006 & -552.5460866006 & -552.5675456006 & -552.4837006006 \\
\hline \multicolumn{7}{c}{2500} \\
\hline Temp. & 2500 & 2600 & 2700 & 2800 & & \\
\hline F6 & -552.6114181838 & -552.6337731838 & -552.6564071838 & -552.6793091838 & -552.7024711838 & -552.7258851838 \\
F5 & -552.6114013912 & -552.6337573912 & -552.6563913912 & -552.6792933912 & -552.7024563912 & -552.7258693912 \\
F4 & -552.6113880470 & -552.6337440470 & -552.6563780470 & -552.6792810470 & -552.7024430470 & -552.7258570470 \\
F3 & -552.6113772336 & -552.6337342336 & -552.6563682336 & -552.6792712336 & -552.7024342336 & -552.7258482336 \\
F2 & -552.6113702498 & -552.6337272498 & -552.6563612498 & -552.6792642498 & -552.7024272498 & -552.7258422498 \\
F1 & -552.6113689953 & -552.6337259953 & -552.6563609953 & -552.6792639953 & -552.7024269953 & -552.7258419953 \\
TS & -552.6113641274 & -552.6337211274 & -552.6563561274 & -552.6792591274 & -552.7024231274 & -552.7258371274 \\
R1 & -552.6113620161 & -552.6337190161 & -552.6563540161 & -552.6792580161 & -552.7024210161 & -552.7258360161 \\
R2 & -552.6113581340 & -552.6337151340 & -552.6563501340 & -552.6792531340 & -552.7024161340 & -552.7258311340 \\
R3 & -552.6113589163 & -552.6337159163 & -552.6563499163 & -552.6792529163 & -552.7024159163 & -552.7258309163 \\
R4 & -552.6113637400 & -552.6337197400 & -552.6563547400 & -552.6792577400 & -552.7024207400 & -552.7258347400 \\
R5 & -552.6113716324 & -552.6337276324 & -552.6563626324 & -552.6792656324 & -552.7024276324 & -552.7258426324 \\
R6 & -552.6113836006 & -552.6337396006 & -552.6563736006 & -552.6792766006 & -552.7024386006 & -552.7258526006 \\
\hline
\end{tabular}
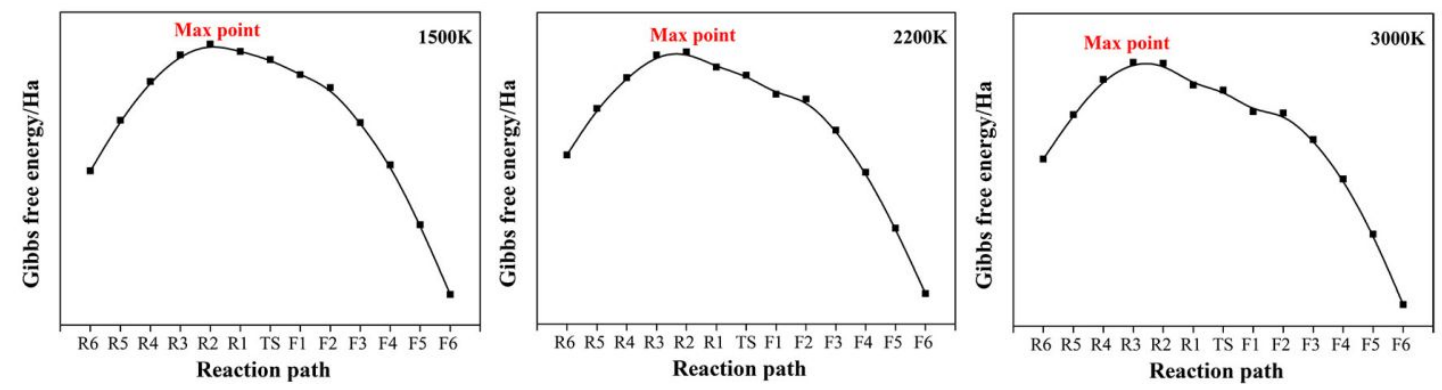

Fig. S2 The maximum Gibbs free energy point at different temperature.

Table S8 The CCSD(T) Gibbs free energy of the geometries.

\begin{tabular}{cc}
\hline Tem.(K) & VTST barrier (kcal/mol) \\
\hline 700 & 65.7095134972 \\
800 & 65.7747744748 \\
900 & 65.8525856404 \\
1000 & 65.9404369565 \\
1100 & 66.0389559323 \\
1200 & 66.1474411147 \\
1300 & 66.2654128820 \\
1400 & 66.3927972902 \\
1500 & 66.5283393206 \\
1600 & 66.6732939920 \\
1700 & 66.8257787762 \\
1800 & 66.9870486920 \\
1900 & 67.1552212112 \\
2000 & 67.3321788619 \\
2100 & 67.5166666256 \\
2200 & 67.7080569926 \\
2300 & 67.9057224536 \\
2400 & 68.1115455368 \\
2500 & 68.3236437140 \\
2600 & 68.5426444946 \\
2700 & 68.7674294471 \\
2800 & 68.9989804157 \\
2900 & 69.2361789688 \\
3000 & 69.4796526160 \\
\hline
\end{tabular}


5 T1 diagnostics for CCSD(T) calculation.

Table S9 T1 diagnostics for all CCSD(T) calculations.

\begin{tabular}{|c|c|c|c|c|}
\hline \multirow{2}{*}{ Species } & \multicolumn{2}{|c|}{ Thiophene } & \multicolumn{2}{|c|}{ Furan } \\
\hline & $\mathbf{T Z}$ & QZ & $\mathbf{T Z}$ & $\mathbf{Q Z}$ \\
\hline TRE & 0.01285 & 0.01296 & 0.01335 & 0.01334 \\
\hline IM1 & 0.01761 & 0.01741 & 0.01554 & 0.0154 \\
\hline IM2 & 0.0151 & 0.01506 & 0.01415 & 0.0141 \\
\hline IM3 & 0.01339 & 0.01351 & 0.01453 & 0.01455 \\
\hline IM4 & 0.01322 & 0.01339 & 0.01337 & 0.01343 \\
\hline IM5 & 0.01145 & 0.01159 & 0.01217 & 0.01229 \\
\hline IM6 & 0.01606 & 0.01601 & 0.01524 & 0.01512 \\
\hline IM7 & 0.01876 & 0.01869 & - & - \\
\hline IM8 & 0.01907 & 0.01996 & - & - \\
\hline IM9 & 0.01318 & 0.01321 & 0.0237 & 0.02323 \\
\hline IM10 & 0.01297 & 0.01319 & 0.0136 & 0.01367 \\
\hline IM11 & 0.01911 & 0.01924 & 0.0194 & 0.01948 \\
\hline IIM & 0.01563 & 0.01551 & 0.01377 & 0.01366 \\
\hline C21-carbene & 0.01706 & 0.01726 & 0.0158 & 0.01584 \\
\hline C23-carbene & 0.02055 & 0.02061 & 0.01816 & 0.01806 \\
\hline C24-carbene & 0.02164 & 0.02161 & 0.01817 & 0.01806 \\
\hline C25-carbene & 0.02133 & 0.02132 & 0.01855 & 0.01839 \\
\hline H-2-QZ & 0.04261 & 0.04272 & 0.03956 & 0.03969 \\
\hline H-3-QZ & 0.04288 & 0.04293 & 0.04028 & 0.0404 \\
\hline H-QZ & 0.0 & 0.0 & 0.0 & 0.0 \\
\hline BIM1 & 0.04307 & 0.04347 & 0.03622 & 0.03637 \\
\hline BIM2 & 0.03069 & 0.03115 & 0.02793 & 0.02813 \\
\hline BIM3 & 0.03977 & 0.04 & 0.03716 & 0.0373 \\
\hline BIM4 & 0.03883 & 0.03922 & 0.0352 & 0.0353 \\
\hline BIM5 & 0.03896 & 0.03937 & 0.03521 & 0.03539 \\
\hline BIM6 & 0.02857 & 0.02881 & 0.03016 & 0.03057 \\
\hline BIM7 & 0.03939 & 0.03968 & 0.03584 & 0.03596 \\
\hline ITS1 & 0.0161 & 0.01625 & 0.02575 & 0.02529 \\
\hline ITS2 & 0.0177 & 0.01781 & 0.01726 & 0.0172 \\
\hline ITS3 & 0.02007 & 0.02016 & 0.01793 & 0.01787 \\
\hline ITS4 & 0.01702 & 0.01717 & 0.01734 & 0.01733 \\
\hline BTS1 & 0.0276 & 0.02773 & 0.02686 & 0.02696 \\
\hline BTS2 & 0.03872 & 0.03898 & 0.03876 & 0.03899 \\
\hline ITS & 0.03591 & 0.03523 & 0.0318 & 0.03149 \\
\hline $\mathrm{TS}$ of $\mathrm{C}_{2,3}-\mathrm{C}_{2,4}$ & 0.01572 & 0.01581 & 0.01685 & 0.01679 \\
\hline $\mathrm{TS}$ of $\mathrm{C}_{2,4}-\mathrm{C}_{2,5}$ & 0.02021 & 0.02023 & 0.01977 & 0.01967 \\
\hline $\mathrm{TS}$ of $\mathrm{C}_{2,5}-\mathrm{C}_{2,1}$ & 0.01792 & 0.01797 & 0.02481 & 0.02461 \\
\hline TS1 & 0.01993 & 0.01972 & 0.01767 & 0.0176 \\
\hline TS2 & 0.02281 & 0.02279 & 0.03362 & 0.03368 \\
\hline TS3 & 0.01996 & 0.01992 & 0.02259 & 0.02244 \\
\hline TS4 & 0.02284 & 0.02236 & 0.02306 & 0.02258 \\
\hline TS5 & 0.01692 & 0.0169 & 0.01622 & 0.01618 \\
\hline TS6 & 0.02608 & 0.02587 & 0.03157 & 0.03132 \\
\hline TS7 & 0.03763 & 0.03677 & 0.04314 & 0.04159 \\
\hline TS8 & 0.05765 & 0.0563 & 0.04746 & 0.04815 \\
\hline TS9 & 0.01986 & 0.01965 & 0.01537 & 0.01521 \\
\hline TS10 & 0.01741 & 0.01741 & 0.01793 & 0.01779 \\
\hline $\mathrm{TS} 11$ & 0.02117 & 0.02121 & 0.02081 & 0.02088 \\
\hline
\end{tabular}




\begin{tabular}{ccccc} 
TS12 & 0.0272 & 0.02695 & 0.03335 & 0.03331 \\
TS13 & 0.03526 & 0.03335 & 0.02762 & 0.02695 \\
TS14 & 0.02359 & 0.02346 & 0.02054 & 0.02035 \\
TS15 & 0.02031 & 0.02014 & - & - \\
TS16 & 0.02677 & 0.02704 & 0.0283 & 0.02836 \\
TS17 & 0.0218 & 0.02158 & - & - \\
TS18 & 0.0218 & 0.02185 & 0.03186 & 0.03187 \\
TS19 & 0.03763 & 0.03689 & 0.02582 & 0.02519 \\
TS20 & 0.01473 & 0.01495 & 0.01427 & 0.01436 \\
TS21 & 0.03050 & 0.03001 & - & - \\
TS22 & 0.01806 & 0.01823 & 0.01782 & 0.01788 \\
TS23 & 0.04074 & 0.0396 & 0.03767 & 0.0367 \\
TS24 & 0.01955 & 0.01957 & 0.01737 & 0.01734 \\
TS25 & 0.14602 & 0.14386 & 0.09918 & 0.09591 \\
TS26 & 0.02353 & 0.02384 & 0.02969 & 0.02988 \\
TS27 & 0.01894 & 0.01864 & 0.01999 & 0.01994 \\
\hline
\end{tabular}

Table S10 Comparison of the MRCI energy with the CCSD(T) energy of some species in thiophene pyrolysis. (unit: $\mathrm{kcal} / \mathrm{mol}$ )

\begin{tabular}{|c|c|c|c|c|c|c|}
\hline Species & $\begin{array}{c}\text { MRCI+Q }^{\mathrm{a}} \\
\text { without ZPE }\end{array}$ & $\begin{array}{c}\mathrm{CCSD}(\mathrm{T})^{\mathrm{b}} \\
\text { without ZPE }\end{array}$ & Deviation & $\begin{array}{l}\text { MRCI+Q }{ }^{\mathrm{a}} \\
\text { with } \mathrm{ZPE}\end{array}$ & $\begin{array}{l}\mathrm{CCSD}(\mathrm{T})^{\mathrm{b}} \\
\text { with ZPE }\end{array}$ & Deviation \\
\hline $\mathrm{C}_{2,1}$-carbene & 81.88 & 83.20 & -1.32 & 81.28 & 80.28 & 1.00 \\
\hline $\mathrm{C}_{2,3}$-carbene & 60.58 & 58.58 & 2.00 & 58.72 & 56.90 & 1.82 \\
\hline $\mathrm{C}_{2,4}$-carbene & 74.31 & 77.75 & -3.44 & 72.38 & 75.39 & -3.01 \\
\hline $\mathrm{C}_{2,5}$-carbene & 55.97 & 55.11 & 0.86 & 55.06 & 53.88 & 1.17 \\
\hline IIM & 69.47 & 67.72 & 1.75 & 68.99 & 65.42 & 3.57 \\
\hline IM10 & 45.93 & 47.16 & -1.23 & 45.38 & 43.67 & 1.71 \\
\hline IM11 & 112.67 & 115.26 & -2.60 & 109.83 & 109.00 & 0.84 \\
\hline IM1 & 48.27 & 47.24 & 1.03 & 43.26 & 45.06 & -1.80 \\
\hline IM2 & 52.04 & 52.51 & -0.47 & 52.89 & 50.30 & 2.59 \\
\hline IM3 & 35.70 & 39.16 & -3.46 & 36.50 & 37.87 & -1.37 \\
\hline IM5 & 49.61 & 50.41 & -0.79 & 50.59 & 48.87 & 1.72 \\
\hline IM6 & 34.13 & 37.02 & -2.88 & 35.02 & 35.32 & -0.30 \\
\hline IM7 & 91.34 & 91.71 & -0.37 & 90.87 & 88.48 & 2.39 \\
\hline IM8 & 83.74 & 82.53 & 1.20 & 75.59 & 79.53 & -3.94 \\
\hline IM9 & 63.34 & 62.47 & 0.87 & 57.73 & 60.14 & -2.41 \\
\hline ITS1 & 90.82 & 88.61 & 2.21 & 87.98 & 84.26 & 3.72 \\
\hline ITS2 & 70.08 & 68.72 & 1.36 & 68.26 & 65.42 & 2.84 \\
\hline ITS3 & 76.20 & 76.14 & 0.06 & 73.32 & 72.75 & 0.57 \\
\hline ITS4 & 89.72 & 90.30 & -0.58 & 87.30 & 86.44 & 0.86 \\
\hline ITS & 100.12 & 102.30 & -2.18 & 93.02 & 98.42 & -5.40 \\
\hline TS11 & 124.34 & 126.64 & -2.31 & 119.91 & 120.68 & -0.77 \\
\hline TS12 & 68.93 & 73.25 & -4.32 & 66.00 & 71.09 & -5.09 \\
\hline TS13 & 89.89 & 95.23 & -5.34 & 86.78 & 91.36 & -4.58 \\
\hline TS14 & 106.70 & 109.13 & -2.42 & 101.90 & 104.13 & -2.22 \\
\hline TS15 & 96.30 & 96.87 & -0.56 & 92.14 & 92.64 & -0.50 \\
\hline TS16 & 124.42 & 128.51 & -4.09 & 118.65 & 122.47 & -3.82 \\
\hline TS17 & 99.10 & 99.04 & 0.07 & 95.09 & 94.83 & 0.26 \\
\hline TS18 & 121.18 & 124.22 & -3.04 & 114.94 & 117.52 & -2.58 \\
\hline TS1 & 106.54 & 104.58 & 1.96 & 98.76 & 99.77 & -1.01 \\
\hline TS20 & 93.71 & 95.05 & -1.34 & 91.07 & 89.10 & 1.98 \\
\hline TS21 & 144.86 & 134.86 & 10.00 & 141.22 & 127.23 & 13.99 \\
\hline TS23 & 133.50 & 136.24 & -2.74 & 126.87 & 128.32 & -1.45 \\
\hline \multirow[t]{2}{*}{ TS24 } & 134.59 & 134.11 & 0.48 & 130.25 & 126.10 & 4.14 \\
\hline & & & 19 & & & \\
\hline
\end{tabular}




\begin{tabular}{ccccccc} 
TS25 & 91.54 & 67.70 & 23.84 & 90.61 & 63.33 & 27.27 \\
TS26 & 119.40 & 123.15 & -3.75 & 113.88 & 117.37 & -3.49 \\
TS27 & 103.42 & 108.11 & -4.69 & 98.26 & 102.68 & -4.42 \\
TS2 & 154.71 & 152.37 & 2.34 & 147.85 & 145.25 & 2.60 \\
TS3 & 90.90 & 93.53 & -2.63 & 90.65 & 91.03 & -0.39 \\
TS4 & 68.14 & 69.34 & -1.20 & 65.47 & 66.48 & -1.01 \\
TS5 & 100.24 & 99.20 & 1.05 & 97.55 & 93.53 & 4.02 \\
TS6 & 102.38 & 99.97 & 2.41 & 95.61 & 96.50 & -0.89 \\
TS8 & 112.48 & 123.95 & -11.47 & 107.75 & 119.85 & -12.11 \\
TS9 & 115.61 & 116.63 & -1.03 & 112.09 & 110.54 & 1.56 \\
TS-C 2 - $\mathrm{C}_{2,4}$ & 92.38 & 90.09 & 2.29 & 90.34 & 86.52 & 3.82 \\
TS-C $_{2,4}-\mathrm{C}_{2,5}$ & 92.51 & 90.93 & 1.58 & 90.31 & 87.34 & 2.97 \\
\hline
\end{tabular}

${ }^{\text {a }}$ Calculated at MRCI+Q(10,9)/cc-pVTZ level using ORCA 4.2. ZPE correction was obtained by performing frequcency calculation at $\operatorname{CASSCF}(10,9) / \mathrm{cc}-\mathrm{pVTZ}$ level using same software.

${ }^{b}$ calculation at CCSD(T)/cc-pVTZ level using Gaussian 09D. ZPE correction was obtained from the frequency result at B3LYP/cc-pVTZ level using same software.

Table S11 Comparison of the MRCI energy with the $\operatorname{CCSD}(T)$ energy of the species in furan pyrolysis. (unit: $\mathrm{kcal} / \mathrm{mol}$ )

\begin{tabular}{|c|c|c|c|c|c|c|}
\hline Species & $\begin{array}{c}\text { MRCI+Q }{ }^{\mathrm{a}} \\
\text { without } \mathrm{ZPE}\end{array}$ & $\begin{array}{c}\mathrm{CCSD}(\mathrm{T})^{\mathrm{b}} \\
\text { without } \mathrm{ZPE}\end{array}$ & Deviation & $\begin{array}{l}\text { MRCI+Q }{ }^{\mathrm{a}} \\
\text { with } \mathrm{ZPE} \\
\end{array}$ & $\begin{array}{l}\text { CCSD(T) } \\
\text { with ZPE }\end{array}$ & Deviation \\
\hline $\mathrm{C}_{2,1}$-carbene & 85.19 & 85.00 & 0.19 & 86.00 & 82.24 & 3.76 \\
\hline $\mathrm{C}_{2,3}$-carbene & 57.02 & 56.44 & 0.58 & 55.10 & 54.47 & 0.63 \\
\hline $\mathrm{C}_{2,4}$-carbene & 76.31 & 75.87 & 0.43 & 73.15 & 72.61 & 0.55 \\
\hline $\mathrm{C}_{2,5}$-carbene & 55.17 & 53.23 & 1.94 & 52.78 & 51.97 & 0.81 \\
\hline IIM & 49.93 & 49.79 & 0.14 & 47.33 & 46.78 & 0.55 \\
\hline IM10 & 37.05 & 34.16 & 2.89 & 31.98 & 32.11 & -0.12 \\
\hline IM11 & 105.87 & 104.96 & 0.91 & 99.76 & 100.00 & -0.24 \\
\hline IM1 & 32.39 & 29.94 & 2.46 & 26.32 & 26.95 & -0.63 \\
\hline IM2 & 35.26 & 33.51 & 1.76 & 31.34 & 30.49 & 0.85 \\
\hline IM3 & 42.76 & 43.07 & -0.31 & 42.77 & 41.14 & 1.63 \\
\hline IM4 & 58.41 & 59.03 & -0.63 & 57.94 & 56.16 & 1.78 \\
\hline IM6 & 16.26 & 17.26 & -1.00 & 14.19 & 14.84 & -0.65 \\
\hline IM9 & 85.92 & 86.38 & -0.46 & 82.79 & 82.26 & 0.53 \\
\hline ITS1 & 91.81 & 91.09 & 0.72 & 85.73 & 85.08 & 0.65 \\
\hline ITS2 & 70.14 & 68.86 & 1.28 & 66.51 & 65.37 & 1.14 \\
\hline ITS3 & 72.80 & 72.55 & 0.25 & 70.91 & 69.33 & 1.58 \\
\hline ITS4 & 90.67 & 90.30 & 0.37 & 86.93 & 86.51 & 0.42 \\
\hline ITS & 86.23 & 88.52 & -2.29 & 83.90 & 84.17 & -0.27 \\
\hline TS10 & 106.93 & 111.07 & -4.14 & 103.42 & 104.04 & -0.62 \\
\hline TS11 & 104.22 & 102.91 & 1.31 & 97.57 & 95.90 & 1.67 \\
\hline TS12 & 67.56 & 69.18 & -1.62 & 64.83 & 66.06 & -1.22 \\
\hline TS13 & 84.51 & 85.53 & -1.02 & 80.19 & 81.14 & -0.95 \\
\hline TS14 & 89.72 & 87.26 & 2.46 & 82.92 & 81.68 & 1.24 \\
\hline TS16 & 113.08 & 110.69 & 2.38 & 102.13 & 104.22 & -2.10 \\
\hline TS18 & 115.68 & 119.51 & -3.83 & 114.18 & 113.39 & 0.79 \\
\hline TS1 & 88.38 & 90.28 & -1.91 & 86.90 & 84.70 & 2.20 \\
\hline TS22 & 104.64 & 106.15 & -1.51 & 100.80 & 100.61 & 0.19 \\
\hline TS23 & 140.25 & 144.22 & -3.96 & 137.26 & 136.73 & 0.53 \\
\hline TS24 & 114.92 & 117.33 & -2.42 & 111.85 & 110.44 & 1.41 \\
\hline TS26 & 110.52 & 109.81 & 0.70 & 103.79 & 103.01 & 0.78 \\
\hline TS27 & 73.06 & 73.67 & -0.61 & 68.86 & 66.96 & 1.89 \\
\hline TS2 & 109.58 & 107.85 & 1.73 & 99.74 & 99.06 & 0.69 \\
\hline TS3 & 95.53 & 98.83 & -3.30 & 95.12 & 95.70 & -0.58 \\
\hline TS4 & 67.22 & 70.70 & -3.47 & 65.48 & 66.95 & -1.47 \\
\hline TS5 & 78.06 & 80.45 & -2.40 & 74.35 & 73.98 & 0.38 \\
\hline TS6 & 108.61 & 106.10 & 2.50 & 101.64 & 102.27 & -0.63 \\
\hline \multicolumn{7}{|c|}{20} \\
\hline
\end{tabular}




\begin{tabular}{ccccccc} 
TS8 & 128.62 & 132.17 & -3.55 & 126.60 & 125.70 & 0.91 \\
TS9 & 81.20 & 77.40 & 3.80 & 72.54 & 70.04 & 2.50 \\
TS-C $2,3-\mathrm{C}_{2,4}$ & 94.54 & 95.62 & -1.08 & 92.23 & 91.63 & 0.60 \\
$\mathrm{TS}_{2,4}-\mathrm{C}_{2,5}$ & 96.35 & 94.02 & 2.32 & 92.11 & 90.10 & 2.01 \\
$\mathrm{TS}_{2, \mathrm{C}_{2,5}-\mathrm{C}_{2,1}}$ & 114.59 & 114.62 & -0.03 & 109.66 & 109.31 & 0.35 \\
\hline
\end{tabular}

${ }^{a}$ Calculated at MRCI+Q(10,9)/cc-pVTZ level using ORCA 4.2. ZPE correction was obtained by performing frequcency calculation at $\operatorname{CASSCF}(10,9) / \mathrm{cc}-\mathrm{pVTZ}$ level using same software.

${ }^{b}$ calculation at CCSD(T)/cc-pVTZ level using Gaussian 09D. ZPE correction was obtained from the frequency result at B3LYP/cc-pVTZ level using same software.

\section{$6 M R C I+Q$ and $C A S S C F$ calculation.}

We have used the complete active space self-consistent field (CASSCF) method [1-3] followed by the multi-reference configuration interaction with Davidson correction $(\mathrm{MRCI}+\mathrm{Q})$ [4]. Singlet and triplet states were computed using the stateaveraged CASSCF (SA-CASSCF) method. The SA-CASSCF molecular orbitals were used for the MRCI+Q calculations. All MRCI+Q and CASSCF calculations were carried out at Aug-cc-pVTZ level using Orca4.2 package [5-6].

\section{Reference}

[1] Roos, B. O.; Taylor, P. R.; et al. A complete active space SCF method (CASSCF) using a density matrix formulated super-CI approach. Chem. Phys., 1980, 48, 157-173.

[2] Siegbahn, P.; Heiberg, A.; et al. A comparison of the super-CI and the newton-raphson scheme in the complete active space SCF method. Phys. Scr., 1980, 21, 323-327.

[3] Siegbahn, P. E.; Almlöf, J.; et al. The complete active space SCF (CASSCF) method in a Newton-Raphson formulation with application to the HNO molecule. J. Chem. Phys., 1981, 74, 2384-2396.

[4] Adams, G. F.; Jensen, J. O.; et al. Ab initio study of the electronic mag- netic circular dichroism spectrum in acetylene: the B-X And 11B2X transitions. J. Chem. Phys., 1991, 94, 1332-1345.

[5] F. Neese. The ORCA program system. Wiley Interdiscip. Rev. Comput. Mol. Sci., 2012, 2, 73-78 .

[6] F. Neese. Software update: the ORCA program system version 4.0. Wiley Interdiscip. Rev. Comput. Mol. Sci., 2017, 8, e1327. 